\title{
On realcompact topological vector spaces
}

\author{
J. Ka̧kol · M. López-Pellicer
}

Received: 7 January 2010 / Accepted: 7 May 2010 / Published online: 3 February 2011

(C) The Author(s) 2011. This article is published with open access at Springerlink.com

\begin{abstract}
This survey paper collects some of older and quite new concepts and results from descriptive set topology applied to study certain infinite-dimensional topological vector spaces appearing in Functional Analysis, including Fréchet spaces, $(L F)$-spaces, and their duals, $(D F)$-spaces and spaces of continuous real-valued functions $C(X)$ on a completely regular Hausdorff space $X$. Especially $(L F)$-spaces and their duals arise in many fields of Functional Analysis and its applications, for example in Distributions Theory, Differential Equations and Complex Analysis. The concept of a realcompact topological space, although originally introduced and studied in General Topology, has been also studied because of very concrete applications in Linear Functional Analysis.
\end{abstract}

Keywords Angelicity · Baire and (b-) Baire-like $\cdot$ Bornological $\cdot$ Borel set $\cdot C^{*}$-embedded . Class $\mathfrak{G} \cdot(D F)$ space $\cdot$ Distinguished space $\cdot$ Fréchet-Urysohn $\cdot k$-Space $\cdot K$-Analytic $\cdot$ (Weakly) Lindelöf $(\Sigma) \cdot$ Locally convex space $\cdot(\Sigma$-)Quasi-Suslin space $\cdot$ (Strongly) realcompact space · (Compact) resolution · Talagrand compact · (Countable) tightness . Trans-separable · Weakly compact · (WCG) space · Web-bounded (compact)

Mathematics Subject Classification (2000) $\quad 54 \mathrm{H} 05 \cdot 46 \mathrm{~A} 04 \cdot 46 \mathrm{~A} 50$

Dedicated to Professor Manuel Valdivia, excellent professor and mathematical researcher, on the occasion of his 80th birthday.

The research for the first named author was (partially) supported by Ministry of Science and Higher Education, Poland, Grant no. NN201 274033 and for the both authors by the project MTM2008-01502 of the Spanish Ministry of Science and Innovation.

J. Ka̧kol (ه)

Faculty of Mathematics and Informatics, A. Mickiewicz University, 61-614 Poznan, Poland

e-mail:kakol@math.amu.edu.pl

M. López-Pellicer

Departamento de Matemática Aplicada and IUMPA, Universidad Politécnica de Valencia, 46022 Valencia, Spain

e-mail: mlopezpe@mat.upv.es 


\section{Introduction}

For a Tichonov space (also named completely regular Hausdorff space) $X$ by $C_{p}(X)$ and $C_{c}(X)$ we denote the space of continuous realvalued maps on $X$ with the pointwise and the compact-open topology, respectively. By $L_{p}(X)$ we denote the $*$-weak dual of $C_{p}(X)$. If $\mathcal{F}:=\{f \in C(X): f(X) \subset[0,1]\}$, then in $[0,1]^{\mathcal{F}}$ the subspace $\{(f(x): f \in \mathcal{F}): x \in X\}$ is homeomorphic to $X$. We identify $X$ with this subspace $\{(f(x): f \in \mathcal{F}): x \in X\}$ and the closure of $X$ in $[0,1]^{\mathcal{F}}$ is the Stone-Čech compactification of $X$, denoted by $\beta X$. Taking into account the restrictions to $\beta X$ of the coordinate projections of $[0,1]^{\mathcal{F}}$ we deduce that each $f \in \mathcal{F}$, and therefore each uniformly bounded $f \in C(X)$ has a unique continuous extension to $\beta X$.

By the realcompactification $v X$ of $X$ we mean the subset of $\beta X$ such that $x \in v X$ if, and only if, each $f \in C(X)$ admits a continuous extension to $X \cup\{x\}$. From regularity it follows that each $f \in C(X)$ admits a continuous extension to $v X$. Therefore the closure in $[0,1]^{C(X)}$ of

$$
\{(f(x): f \in C(X)): x \in X\}
$$

is homeomorphic to $v X$.

By definition $X$ is called realcompact if $X=v X$. From the continuity of the coordinate projections it follows that $X$ is realcompact if, and only if, $X$ is homeomorphic to a closed subspace of a cartesian product of real lines. Every metric separable space is realcompact. Clearly closed subspaces of a realcompact space are realcompact and also each product of realcompact spaces is realcompact. The intersection of a family of realcompact subspaces of a space is realcompact, because this intersection is homeomorphic to the diagonal of a product.

The following well-known characterization of realcompact spaces will be used in the sequel, see $[16,28]$.

Proposition 1 A completely regular Hausdorff space $X$ is realcompact if, and only if, for every element $x \in \beta X \backslash X$ there exists $h \in C(\beta X), h(X) \subset] 0,1]$, i.e. which is positive on $X$ and $h(x)=0$.

Proof Assume that the condition holds. Then

$$
\left.\left.X=\bigcap\left\{h_{y}^{-1}\right] 0,1\right]: y \in \beta X \backslash X\right\} .
$$

As each $\left.\left.h_{y}^{-1}\right] 0,1\right]$ is a realcompact subspace of $\beta X$ (since $\left.\left.h_{y}^{-1}\right] 0,1\right]$ is homeomorphic to

$$
(\beta X \times] 0,1]) \cap G\left(h_{y}\right),
$$

where $G\left(h_{y}\right)$ means the graph of $\left.h_{y}\right)$, then $X$ is also realcompact. Conversely, if $X$ is realcompact and

$$
x_{0} \in \beta X \backslash X=\beta X \backslash v X,
$$

then there exists a continuous function $f: X \rightarrow \mathbb{R}$ which cannot be extended continuously to $X \cup\left\{x_{0}\right\}$. From

$$
f(x)=\max (f(x), 0)+\min (f(x), 0)=1+\max (f(x), 0)-(1-\min (f(x), 0))
$$

we know that one of the functions $g_{1}(x)=1+\max (f(x), 0)$ or $g_{2}(x)=1-\min (f(x), 0)$ cannot be extended continuously to $X \cup\left\{x_{0}\right\}$. So $\left(^{*}\right)$ there exists a continuous function 
$g: X \rightarrow\left[1, \infty\left[\right.\right.$ which cannot be extended continuously to $X \cup\left\{x_{0}\right\}$. Let $\widehat{h}$ be a continuous extension of the bounded function $h:=1 / g$ to $\beta X$. If $\widehat{h}\left(x_{0}\right) \neq 0$, then we get a contradiction with (*). Hence $\widehat{h}\left(x_{0}\right)=0$.

Recall that a uniform space $X$ is called trans-separable [31,33] if every uniform cover of $X$ has a countable subcover. Separable uniform spaces and Lindelöf uniform spaces are transseparable; the converse is not true in general although every trans-separable pseudometric space is separable.

Clearly a uniform space is trans-separable if, and only if, it is uniformly isomorphic to a subspace of a uniform product of separable pseudometric spaces. This implies that every uniform quasi-Suslin space [71, Chapter 1, Sect. 4.2] is trans-separable. Note also that transseparable spaces enjoy good permanence properties. In particular, the class of trans-separable spaces is hereditary, productive and closed under uniform continuous images, see [56].

For a topological vector space (tvs, in brief) $E$ the trans-separability means that $E$ is isomorphic to a subspace of the product of metrizable separable tvs. Thus, in particular if $E$ is a locally convex space (lcs, in brief), then the weak dual $\left(E^{\prime}, \sigma\left(E^{\prime}, E\right)\right)$ of $E$ is trans-separable.

It is easy to see that a tvs $E$ is trans-separable if, and only if, for every neighborhood of zero $U$ in $E$ there exists a countable subset $N$ of $E$ such that $E=N+U$, see for example [30,46,57,58].

Also a tvs $E$ is trans-separable if, and only if, for each continuous F-seminorm $p$ on $E$ the F-seminormed space $(E, p)$ is separable, or the associated F-normed space $E / \operatorname{ker} p$ is separable.

The concept of trans-separable spaces has been used to study several problems both from analysis and topology, for example while studying the metrizability of precompact sets in uniform spaces and in the class of lcs, we refer the reader to papers [12,15,21,22,40,41,58, 64]. Pfister [57] proved the following:

Proposition 2 A lcs E is trans-separable if, and only if, for every neighborhood of zero $U$ in $E$ its polar $U^{\circ}$ is $\sigma\left(E^{\prime}, E\right)$-metrizable.

This fact has been applied by Pfister [57] to show that precompact sets in $(D F)$-spaces are metrizable.

Note the following link between realcompact and trans-separable space, see also related results of this of type in [32].

Proposition 3 A completely regular topological Hausdorff space is realcompact if, and only if, there exists an admissible uniformity $\mathcal{N}$ on $X$ such that $(X, \mathcal{N})$ is trans-separable and complete.

Proof If $X$ is realcompact, then it is homeomorphic to a closed subset of $\mathbb{R}^{C(X)}$. Then the induced uniformity in $X$ is admissible complete and trans-separable. Conversely, if $\mathcal{N}$ is a trans-separable, complete admissible uniformity on $X$, then $(X, \mathcal{N})$ is isomorphic to a closed subspace of a product of metrizable separable (by trans-separability) uniform spaces. Therefore $X$ is realcompact.

Proposition 1 may suggest the following concept which originally has been introduced by Ka̧kol and Śliwa [39].

Definition 1 We shall say that $X$ is strongly realcompact if for every sequence $\left(x_{n}\right)_{n}$ of elements in $\beta X \backslash X$ there exists $f \in C(\beta X)$ which is positive on $X$ and vanishes on some subsequence of $\left(x_{n}\right)_{n}$. 
Clearly every strongly realcompact space is realcompact. It is known [78, Exer. 1B. 4], that if $X$ is locally compact $\sigma$-compact, then $\beta X \backslash X$ is a zero set in $\beta X$, so $X$ is strongly realcompact. Recall also that a subset $A \subset X$ is said to be $C$-embedded ( $\left(C^{*}\right)$-embedded) if every real-valued continuous (bounded and continuous) function on $A$ can be extended to a continuous function on the whole space $X$.

For strongly realcompact spaces we note the following property. The proof presented below from [39] uses an argument of Negrepontis [51] concerning [27, Theorem 2.7].

Proposition 4 If $X$ is strongly realcompact, then every infinite subset $D$ of $\beta X \backslash X$ contains an infinite subset $S$ which is relatively compact in $\beta X \backslash X$ and $C^{*}$-embedded in $\beta X$.

Proof Let $\left(x_{n}\right)_{n}$ be an injective sequence in $D$ (i.e. $x_{n} \neq x_{m}$ if $n \neq m$ ) and let $f: \beta X \rightarrow$ $[0,1]$ be a continuous function which is positive on $X$ and vanishes on a subsequence of $\left(x_{n}\right)_{n}$. Set

$$
S=\left\{x_{n}: n \in \mathbb{N}\right\} \cap f^{-1}\{0\}, \quad Y_{n}=\left\{x \in \beta X:|f(x)| \geq n^{-1}\right\}, \quad n \in \mathbb{N},
$$

and

$$
X_{1}=S \cup \bigcup_{n} Y_{n}
$$

Note that the space $X_{1}$ is regular and $\sigma$-compact. Hence it must be a normal space. But since $S$ is closed in $X_{1}$, then so it is $C^{*}$-embedded in $X_{1}$. Therefore $S$ is $C^{*}$-embedded in $\beta X_{1}$. But $X \subset X_{1} \subset \beta X$. This yields the equality $\beta X_{1}=\beta X$.

This fact implies that if $X$ is a strongly realcompact space, then every infinite closed subset of $\beta X \backslash X$ contains a copy of the space $\beta \mathbb{N}$.

On the other hand, [4, Example 1.11] Baumgartner and van Douwen provided a separable first countable locally compact realcompact space $X$ (hence strongly realcompact by Theorem 1 below) for which $\beta X \backslash X$ contains a discrete countable subset which is not $C^{*}$-embedded in $\beta X$. This result with [4, Theorem 1.2] can be used to distinguish an example of a locally compact realcompact space $X$ such that $\beta X \backslash X$ contains a sequence $\left(x_{n}\right)_{n}$ for which does not exist $f \in C(\beta X)$ which is positive on $X$ and vanishes on $\left(x_{n}\right)_{n}$. This space provides an example of a locally compact realcompact space which is not strongly realcompact.

The space $\mathbb{Q}$ of rational numbers is not strongly realcompact but applying [14] one gets that $\beta \mathbb{Q} \backslash \mathbb{Q}$ is a $\beta \omega$-space, i.e. if $D$ is a countable discrete subset of $\beta \mathbb{Q} \backslash \mathbb{Q}$ and $\bar{D}$ (the closure in $\beta \mathbb{Q} \backslash \mathbb{Q}$ ) is compact, then $\bar{D}=\beta D$, so $D$ is $C^{*}$-embedded in $\beta \mathbb{Q}$. It is well-known [28] that $\beta \mathbb{Q} \backslash \mathbb{Q}$ contains a countable subset which is not $C^{*}$-embedded in $\beta \mathbb{Q}$.

A filter (filterbasis) $\mathcal{F}$ on a topological space $X$ is said to be unbounded if there exists a continuous real-valued function $f$ on $X$ which is unbounded on each element of $\mathcal{F}$. Then $f$ is said to be unbounded on $\mathcal{F}$.

The following general Theorem 1 below was obtained in [39], parts 1, 2, and [70] part 3. To get property 3 from Theorem 1 we need the following two lemmas.

Lemma 1 A filter $\mathcal{F}$ on a topological space $X$ is unbounded if, and only if, there exists $x \in \bigcap_{F \in \mathcal{F}} \bar{F} \backslash v X$, where the closure is taken in $\beta X$.

Proof Set

$$
K:=\bigcap_{F \in \mathcal{F}} \bar{F}
$$


and assume by contradiction that $K \subset v X$. But then for each continuous real-valued function $f$ on $X$ there exists an open $U_{f} \subset \beta X$ such that $K \subset U_{f}$ and $f \mid U_{f} \cap X$ is bounded. Note that there exists $F \in \mathcal{F}$ contained in $U_{f}$. Indeed, otherwise the family of sets

$$
\left\{\bar{F} \backslash U_{f}: F \in \mathcal{F}\right\}
$$

satisfies the finite intersection property which leads a point in $K \backslash U_{f}$. This is a contradiction.

Hence we proved that there exists $F \in \mathcal{F}$ which belongs to $U_{f}$. This shows that $\mathcal{F}$ is not unbounded.

To prove the converse assume that there exists $x \in K \backslash v X$. Since

$$
v X=\bigcap_{f \in C(X)} v_{f}(X),
$$

where

$$
v_{f}(X):=\left\{x \in \beta X: f^{\beta}(x) \neq \infty\right\},
$$

[28, Problem 8B.3], then there exists $f \in C(X)$ whose extension $f^{\beta}: \beta X \rightarrow \mathbb{R}_{\infty}$, where $\mathbb{R}_{\infty}:=\mathbb{R} \cup\{\infty\}$ (the Alexandroff one-point compactification), has property that $f^{\infty}(x)=$ $\infty$. But $x \in \bar{F}$ for each $F \in \mathcal{F}$, so this proves that $f$ is unbounded on $\mathcal{F}$.

Lemma 2 Each unbounded filterbasis $\mathcal{F}$ on a topological space $X$ is contained in an unbounded ultrafilter $\mathcal{U}$ on $X$.

Proof If $\mathcal{M}:=\{M \subset X: \exists F \in \mathcal{F} ; F \subset M\}$, then $\mathcal{M}$ is an unbounded filter on $X$. By Lemma 1 there exists

$$
x \in \bigcap_{F \in \mathcal{M}} \bar{F} \backslash v X .
$$

Let $\mathcal{A}$ be the family of all filters $\mathcal{G}$ on $X$ containing $\mathcal{M}$ and such that $x \in \bigcap_{F \in \mathcal{G}} \bar{F}$. Ordering $\mathcal{A}$ by inclusion, and since there exists a maximal chain in $\mathcal{A}$ its union $\mathcal{U}$ is an ultrafilter on $X$ containing $\mathcal{F}$ such that

$$
x \in \bigcap_{F \in \mathcal{U}} \bar{F} .
$$

Using Lemma 1 one gets that $\mathcal{U}$ is unbounded on $X$.

Clearly realcompact spaces of pointwise countable type need not be locally compact spaces as the space $\mathbb{R}^{\mathbb{N}}$ shows, Example 1 below. Recall that a topological space $X$ is of pointwise countable type [2] if each $x \in X$ is contained in a compact set $K \subset X$ of countable character in $X$.

For strongly realcompact spaces the situation is different. Now we are ready to prove the following characterization of strongly realcompact spaces.

Theorem 1 1. A topological space $X$ is strongly realcompact if, and only if, it is realcompact and $\beta X \backslash X$ is countably compact. Hence every locally compact realcompact space is strongly realcompact.

2. Every strongly realcompact space of pointwise countable type is locally compact.

3. A realcompact space $X$ is strongly realcompact if, and only if, for each sequence $\left(\mathcal{F}_{n}\right)_{n}$ of unbounded filters (filter bases) there exists a continuous real-valued function on $X$ and a subsequence $\left(\mathcal{F}_{n_{k}}\right)_{k}$ such that $f$ is unbounded on each $\mathcal{F}_{n_{k}}$. 
Proof 1 Assume that $X$ is a strongly realcompact space. Let $P \subset \beta X \backslash X$ be an infinite set and let $\left(x_{n}\right)_{n}$ be an injective sequence in $P$. There exists a continuous function

$$
f: \beta X \rightarrow[0,1]
$$

which is positive on $X$ and zero on some subsequence $\left(x_{k_{n}}\right)_{n}$ of $\left(x_{n}\right)_{n}$. Then we note

$$
\left\{x_{k_{n}}: n \in \mathbb{N}\right\} \subset f^{-1}(0) \subset X^{*} .
$$

Hence

$$
\left\{x_{k_{n}}: n \in \mathbb{N}\right\}^{d} \subset f^{-1}(0) .
$$

Note also that $\left\{x_{k_{n}}: n \in \mathbb{N}\right\}^{d}$ is non-empty, where $A^{d}$ is the set of all accumulation points of $A$. This shows that $P$ has an accumulation point.

Now we prove the converse. Assume that $X$ is realcompact and every infinite subset of $\beta X \backslash X$ has an accumulation point in $\beta X \backslash X$.

Let $\left(x_{n}\right)_{n}$ be a sequence in $\beta X \backslash X$. If $P=\left\{x_{n}: n \in \mathbb{N}\right\}$ is finite, then the realcompact property of $X$ implies that there exists a continuous function $f: \beta X \rightarrow[0,1]$ which is positive on $X$ and zero on a subsequence of $\left(x_{n}\right)_{n}$. If $P=\left\{x_{n}: n \in \mathbb{N}\right\}$ is infinite, take $p \in P^{d} \backslash X$. Then there exists a continuous function

$$
f: \beta X \rightarrow[0,1]
$$

which is positive on $X$ and vanishes on $p$. Note that for every $r>0$ the set $P \cap f^{-1}([0, r))$ is infinite, since $f^{-1}([0, r))$ is a neighbourhood of the point $p \in P^{d}$. Let us consider two possible cases.

(1) The set $P \cap f^{-1}(0)$ is infinite. In that case $f$ is positive on $X$ and zero on some subsequence of the sequence $\left(x_{n}\right)_{n}$.

(2) The set $P \cap f^{-1}(0)$ is finite. Since for every $r>0$ the set $P \cap f^{-1}([0, r))$ is infinite, then there exists an injective sequence $\left(t_{n}\right)_{n}$ in $P$ such that the sequence $\left(f\left(t_{n}\right)\right)_{n}$ is strictly decreasing and converges to zero. Let $P_{0}=\left\{t_{n}: n \in \mathbb{N}\right\}, s_{0}=1$ and $s_{k} \in\left(f\left(t_{k+1}\right), f\left(t_{k}\right)\right)$ for all $k \in \mathbb{N}$. Then the sequence $\left(s_{k}\right)_{k}$ is decreasing and converges to zero. Set

$$
F_{k}=f^{-1}\left(\left[s_{k}, s_{k-1}\right]\right)
$$

for $k \in \mathbb{N}$. Then $F_{k}$ is compact and $t_{k} \in F_{n}$ if, and only if, $k=n$. Moreover,

$$
X \subset f^{-1}((0,1])=\bigcup_{k} F_{k}
$$

and

$$
P_{0} \cap F_{k}=\left\{t_{k}\right\}, \quad k \in \mathbb{N} .
$$

If $f(x)=c>0$, then $x \in f^{-1}\left(\left(2^{-1} c, 1\right]\right)$. Since $f\left(t_{k}\right) \rightarrow 0$, one gets that $x \notin P_{0}^{d}$. Hence, if $x \in P_{0}^{d}$, then $x \in f^{-1}(0)$. Hence $x \notin \bigcup_{k} F_{k}$. We showed that

$$
P_{0}^{d} \cap\left(\bigcup_{k} F_{k}\right)=\varnothing .
$$

But $X$ is realcompact. Hence for every $k \in \mathbb{N}$ there exists a continuous function $f_{k}: \beta X \rightarrow$ $[0,1]$ which is positive on $X$ and zero on $t_{k}$. Next set

$$
T_{n}^{k}=f_{k}^{-1}\left(\left[n^{-1}, 1\right]\right), \quad n, k \in \mathbb{N} .
$$


Then

$$
X \subset f_{k}^{-1}((0,1])=\bigcup_{n} T_{n}^{k}
$$

and $t_{k} \notin T_{n}^{k}$ for all $k, n \in \mathbb{N}$. Moreover

$$
X \subset \bigcup_{k} F_{k} \cap X \subset \bigcup_{k} \bigcup_{n} F_{k} \cap T_{n}^{k}, \quad P_{0} \cap\left(F_{k} \cap T_{n}^{k}\right) \subset P_{0} \cap F_{k}=\left\{t_{k}\right\} .
$$

But $t_{k} \notin F_{k} \cap T_{n}^{k}$, so

$$
P_{0} \cap\left(F_{k} \cap T_{n}^{k}\right)=\varnothing
$$

for all $n, k \in \mathbb{N}$. Hence $P_{0} \cap W=\emptyset$ and

$$
P_{0}^{d} \cap W \subset P_{0}^{d} \cap \bigcup_{k} F_{k}=\emptyset,
$$

where $W=\bigcup_{k, n} F_{k} \cap T_{n}^{k}$. Therefore we have $\overline{P_{0}} \cap W=\emptyset$. We showed that there exists an infinite subset $P_{0}$ of $P$ and an infinite sequence of compact sets $\left(K_{n}\right)_{n}$ such that

$$
X \subset \bigcup_{n} K_{n} \subset \beta X, \quad\left(\bigcup_{n} K_{n}\right) \cap \overline{P_{0}}=\emptyset .
$$

For every $n \in \mathbb{N}$ let $g_{n}: \beta X \rightarrow[0,1]$ be a continuous function such that

$$
g_{n}\left|K_{n}=1, \quad g_{n}\right| \overline{P_{0}}=0 .
$$

Put

$$
g=\sum_{n} 2^{-n} g_{n}
$$

The function $g: \beta X \rightarrow[0,1]$ is continuous, positive on $X$ and zero on some subsequence of the sequence $\left(x_{n}\right)_{n}$. This shows that for every sequence $\left(x_{n}\right)_{n}$ in $\beta X \backslash X$ there exists a continuous function on $\beta X$ which is positive on $X$ and vanishes on some subsequence of $\left(x_{n}\right)_{n}$.

2 Assume that $X$ is a strongly realcompact space of pointwise countable type but $X$ is not locally compact. Then there exist $x_{0} \in X$ for which does not exist a relatively compact neighbourhood but for $x_{0}$ there exists a compact set $K$ with $x_{0} \in K$ and which admits a countable (decreasing) basis $\left(U_{n}\right)_{n}$ of neighbourhoods of $K$. For every $n \in \mathbb{N}$ choose $x_{n} \in\left(\overline{U_{n}} \backslash X\right)$, where the closure is taken in $\beta X$. Note that

$$
(\beta X \backslash K) \cap\left\{x_{n}\right\}^{d}=\emptyset .
$$

Indeed, let $x \in(\beta X \backslash K)$. Let $V \subset \beta X$ be an open neighbourhood of $K$ such that $x \in(\beta X \backslash \bar{V})$. Then there exists $n_{0} \in \mathbb{N}$ such that $U_{n_{0}} \subset V \cap X$, so $\overline{U_{n_{0}}} \subset \bar{V}$. Since

$$
\left\{x_{n}\right\}^{d} \subset \overline{U_{n_{0}}} \subset \bar{V}
$$

then one gets that $x \in \beta X \backslash\left\{x_{n}\right\}^{d}$. Hence $\left\{x_{n}\right\}^{d} \subset K$. Clearly $\left\{x_{n}\right\}^{d}$ is non-empty. This shows however that $X$ is not strongly realcompact. A contradiction.

3 Assume that $X$ is strongly realcompact and that each $\mathcal{F}_{n}$ is an unbounded filterbasis on $X$. For each $n \in \mathbb{N}$ there exists an accumulation point of $\mathcal{F}_{n}$, say $x_{n} \in \beta X \backslash v X$. Since $X$ is a realcompact space, then $v X=X$. But $X$ is strongly realcompact, so there exists a subsequence $\left(x_{n_{k}}\right)_{k}$ of $\left(x_{n}\right)_{n}$ and a positive continuous function $g \in C(X)$ such that $g(x) \leq 1$ 
and $g^{\beta}\left(x_{n_{k}}\right)=0$ for all $k \in \mathbb{N}$. But then $f:=g^{-1} \in C(X)$. Hence $f$ is unbounded on each $\mathcal{F}_{n_{k}}$ since

$$
x_{n_{k}} \in \bigcap_{F \in \mathcal{F}_{n_{k}}} \bar{F} \backslash v_{f}(X) .
$$

This proves one direction of the statement 3 .

To prove the converse assume that $\left(x_{n}\right)_{n}$ is a sequence in $\beta X \backslash X$. Then for each $n \in \mathbb{N}$ there exists a filter $\mathcal{F}_{n}$ on $X$ which converges to $x_{n}$ in the space $\beta X$. But

$$
x_{n} \in \bigcap_{F \in \mathcal{F}_{n}} \bar{F}, \quad x_{n} \notin X=v X .
$$

This shows that each $\mathcal{F}_{n}$ is unbounded on $X$. But by the assumption there exists a subsequence $\left(\mathcal{F}_{n_{k}}\right)_{k}$ of $(\mathcal{F})_{n}$ and $f \in C(X)$ which is unbounded on each $\mathcal{F}_{n_{k}}$. Set

$$
g(x):=(1+|f(x)|)^{-1}
$$

for each $x \in X$. Clearly the function $g$ is positive on $X$ and is continuous and bounded. Therefore there exists a continuous extension $g^{\beta}$ of $g$ to $\beta X$ and clearly $g^{\beta}\left(x_{n_{k}}\right)=0$. This proves that $X$ is strongly realcompact. The proof is completed.

In [39] we presented the following example of a strongly realcompact space which is not locally compact.

Example 1 There is a strongly realcompact space not locally compact. The space $\mathbb{R}^{\mathbb{N}}$ is realcompact and it is not strongly realcompact.

Proof Let $P$ be a countably and non-empty subset of $\beta \mathbb{N} \backslash \mathbb{N}$. Note that the subspace $X:=$ $\mathbb{N} \cup P$ of $\beta \mathbb{N}$ is a Lindelöf space. Hence it is a realcompact space. On the other hand, since every countably and closed subset of $\beta \mathbb{N}$ is finite, [78, p. 71], one gets that the space $\beta X \backslash X=(\beta \mathbb{N} \backslash \mathbb{N}) \backslash P$ is countably compact. On the other hand $X$ is not locally compact. Now Theorem 1 applies to deduce that $X$ is strongly realcompact. The second statement follows directly from Theorem 1 .

The following theorem from [39] describes strongly realcompact spaces in term of $C(X)$ and applies to characterize bornological and Baire-like spaces $C_{c}(X)$ for locally compact spaces $X$. Recall that a lcs $E$ is Baire-like [60] if for every increasing sequence $\left(A_{n}\right)_{n}$ of absolutely convex closed sets covering $E$ there exists $m \in \mathbb{N}$ such that $A_{m}$ is a neighbourhood of zero in $E$.

Theorem 2 (i) If $X$ is a strongly realcompact space, then $C_{c}(X)$ is Baire-like and bornological.

(ii) Consequently if $X$ is locally compact, then $C_{c}(X)$ is Baire-like and bornological if, and only if, $X$ is realcompact.

(iii) Let $X$ be a space of pointwise countable type. Then $C_{c}(X)$ is bornological and Bairelike if, and only if, $X$ is strongly realcompact.

We recall a few concepts which will be used in the sequel.

We shall say that $X$ admits a compact resolution if there is a family $\left\{K_{\alpha}: \alpha \in \mathbb{N}^{\mathbb{N}}\right\}$ of compact sets covering $X$ with $K_{\alpha} \subset K_{\beta}$ if $\alpha \leq \beta$. If $X$ is a lcs and $K_{\alpha}$ are bounded, i.e. absorbed by neighbourhoods of zero of $X$, then $\left\{K_{\alpha}: \alpha \in \mathbb{N}^{\mathbb{N}}\right\}$ is called a bounded resolution. 
(a) A topological space $X$ is a Lindelöf $\Sigma$-space if there is an upper semi-continuous (usco) map from a (nonempty) subset $\Sigma \subset \mathbb{N}^{\mathbb{N}}$ with compact values in $X$ whose union is $X$, where the set of integers $\mathbb{N}$ is discrete and $\mathbb{N}^{\mathbb{N}}$ has the product topology $[2,45,49,69]$. If the same holds for $\Sigma=\mathbb{N}^{\mathbb{N}}$, then $X$ is called $K$-analytic.

(b) $X$ is $\Sigma$-quasi-Suslin if there exists a set-valued map $T$ from $\Sigma \subset \mathbb{N}^{\mathbb{N}}$ into $X$ covering $X$ such that if $\alpha_{n} \rightarrow \alpha$ in $\Sigma$ and $x_{n} \in T\left(\alpha_{n}\right)$, then $\left(x_{n}\right)_{n}$ has a cluster point in $T(\alpha)$. If $\Sigma=\mathbb{N}^{\mathbb{N}}$, then $X$ is called a quasi-Suslin space, [71].

Note that half of Proposition 5 below is Lemma 29 of [19].

Proposition 5 If $X$ is $\Sigma$-quasi-Suslin (quasi-Suslin), then $v X$ is Lindelöf $\Sigma$ (K-analytic). Hence $X$ is Lindelöf $\Sigma$ if, and only if, $X$ is Lindelöf and $\Sigma$-quasi-Suslin.

Proof Let $T$ be a map on $\Sigma \subset \mathbb{N}^{\mathbb{N}}$ as in (b). Every $T(\alpha)$ is countably compact, so its closure $\overline{T(\alpha)}$ in $v X$ is compact. The map

$$
\alpha \rightarrow \overline{T(\alpha)}
$$

is (usco), so

$$
Z:=\bigcup_{\alpha \in \Sigma} \overline{T(\alpha)}
$$

is Lindelöf $\Sigma$. Then $Z$ is Lindelof and therefore $Z=v Z$. Since

$$
X \subset Z \subset v X,
$$

then $Z=v Z=v X$ is Lindelöf $\Sigma$. The other case goes similarly for $\Sigma=\mathbb{N}^{\mathbb{N}}$.

(c) $X$ is web-compact [52] if there exists a nonempty subset $\Sigma \subset \mathbb{N}^{\mathbb{N}}$ and a family $\left\{A_{\alpha}\right.$ : $\alpha \in \Sigma\}$ in $X$ such that if

$$
C_{n_{1}, \ldots, n_{k}}:=\bigcup\left\{A_{\beta}: \beta=\left(m_{k}\right) \in \Sigma, m_{j}=n_{j}, j=1, \ldots, k\right\}
$$

for $\alpha=\left(n_{k}\right) \in \Sigma$, then

$$
\overline{\bigcup\left\{A_{\alpha}: \alpha \in \Sigma\right\}}=X,
$$

and if $\alpha=\left(n_{k}\right) \in \Sigma$ and $x_{k} \in C_{n_{1}, n_{2}, \ldots, n_{k}}$, then $\left(x_{k}\right)_{k}$ has a cluster point in $X$. All $\Sigma$-quasi-Suslin spaces are web-compact. By [52, Theorem 3] the space $C_{p}(X)$ is angelic if $X$ is web-compact.

(d) $X$ is web-bounding [52] if

$$
X=\overline{\bigcup\left\{A_{\alpha}: \alpha \in \Sigma\right\}}
$$

such that if $\alpha=\left(n_{k}\right) \in \Sigma$ and $x_{k} \in C_{n_{1}, n_{2}, \ldots, n_{k}}$, then $\left(x_{k}\right)_{k}$ is i.e., $\left(f\left(x_{k}\right)\right)_{k}$ is bounded for each $f$ in $C(X)$. If $X=\bigcup\left\{A_{\alpha}: \alpha \in \Sigma\right\}$, then $X$ is called strongly web-bounding.

(e) A lcs $E$ is web-bounded if $E$ is covered by a family $\left\{A_{\alpha}: \alpha \in \Sigma\right\}$ of sets for $\Sigma \subset \mathbb{N}^{\mathbb{N}}$ such that if $\alpha=\left(n_{k}\right) \in \Sigma$ and

$$
x_{k} \in C_{n_{1}, n_{2}, \ldots, n_{k}},
$$

then $\left(x_{k}\right)_{k}$ is bounded. Since $A_{\alpha} \subset C_{n_{1}, n_{2}, \ldots, n_{k}}, k \in \mathbb{N}$, then $A_{\alpha}$ are bounded. If $X$ is $\sigma$-bounded, i.e. covered by a sequence $\left(B_{k}\right)_{k}$ of bounding sets, then $C_{p}(X)$ has a bounded resolution. Indeed, for $\alpha=\left(n_{k}\right) \in \mathbb{N}^{\mathbb{N}}$ set

$$
A_{\alpha}=\bigcap_{k}\left\{f \in C(X): \sup _{x \in B_{k}}|f(x)| \leq n_{k}\right\} \text {. }
$$


In [20] we proved that a cosmic space $X$ is $\sigma$-compact if, and only if, $C_{p}(X)$ has a bounded resolution.

Clearly $K$-analytic $\Rightarrow$ Lindelöf $\Sigma \Rightarrow$ Lindelöf $\Rightarrow$ realcompact. Every $K$-analytic space has a compact resolution [66]; the converse fails [66].

The following implications below are obvious:

Quasi-Suslin or Lindelöf $\Sigma \Longrightarrow \Sigma$-quasi-Suslin $\Longrightarrow$ web-compact.

The product $\mathbb{R}^{\mathbb{R}}$ (as separable) provides a concrete example of a web-compact space which is not $\Sigma$-quasi-Suslin, since $\mathbb{R}^{\mathbb{R}}$ is realcompact but not Lindelöf and Proposition 5 above applies. There exist however $\Sigma$-quasi-Suslin (even quasi-Suslin) spaces which are not Lindelöf $\Sigma$-spaces, see Remark 1 below.

In [35] we proved the following theorem describing Lindelöf $\Sigma$-spaces $v X$. Note that the equivalence ii $\Leftrightarrow$ iii is essentially Theorem 3 and Proposition 9 from [19].

Theorem 3 For a Tichonov space $X$ the following assertions are equivalent:

(i) $X$ is strongly web-bounding.

(ii) $v X$ is a Lindelöf $\Sigma$-space

(iii) $C_{p}(X)$ is web-bounded.

(iv) $L_{p}(X)$ is web-bounded.

(v) $C_{p}(X)$ is a dense subspace of a lcs which is a Lindelöf $\Sigma$-space.

(vi) $L_{p}(v X)$ is a Lindelöf $\Sigma$-space.

(vii) $L_{p}(v X)$ is $\Sigma$-quasi-Suslin..

We note also the following fact from [35].

Proposition 6 Every lcs with a bounded resolution is web-bounded and every web-bounded $C_{p}(X)$ is angelic.

Proof The first claim is clear. By Theorem 3 the space $v X$ is Lindelöf $\Sigma$. Since then $v X$ is web-compact, then $C_{p}(v X)$ is angelic [52, Theorem 2] and $C_{p}(X)$ is also angelic [12, Note 4].

\section{Locally convex spaces in class $\mathfrak{G}$}

This section deals with the same problems studied in the previous part but in a more general setting. First we recall the concept of the class $\mathfrak{G}$.

Following Cascales and Orihuela [12] a lcs $E$ is said to be in class $\mathfrak{G}$ if there is a family $\left\{A_{\alpha}: \alpha \in \mathbb{N}^{\mathbb{N}}\right\}$ of subsets of its topological dual $E^{\prime}$ (called its $\mathfrak{G}$-representation) such that:

(a) $E^{\prime}=\bigcup\left\{A_{\alpha}: \alpha \in \mathbb{N}^{\mathbb{N}}\right\}$,

(b) $A_{\alpha} \subset A_{\beta}$ when $\alpha \leq \beta$,

(c) in each $A_{\alpha}$, sequences are equicontinuous,

Condition (c) implies that every set $A_{\alpha}$ is $\sigma\left(E^{\prime}, E\right)$-relatively countably compact. Therefore the weak dual $\left(E^{\prime}, \sigma\left(E^{\prime}, E\right)\right)$ of a lcs $E$ in class $\mathfrak{G}$ has a relatively countably compact resolution. The class $\mathfrak{G}$ contains (among the others) $(L M)$-spaces (hence $(L F)$-spaces), the dual metric spaces (hence $(D F)$-spaces), the space of distributions $D^{\prime}(\Omega)$ and the space $A(\Omega)$ of the real analytic functions for open $\Omega \subset \mathbb{R}^{\mathbb{N}}$, see e.g. [8,25].

The class $\mathfrak{G}$ is stable by taking subspaces, separated quotients, completions, countable direct sums and countable products [12].

Recall the following properties concerning spaces in class $\mathfrak{G}$. 
Proposition 7 (i) Let $\left(E_{n}\right)_{n}$ be a sequence of lcs in class $\mathfrak{G}$. Then the topological direct sum $E:=\bigoplus_{n} E_{n}$ belongs to class $\mathfrak{G}$.

(ii) Let $\left(E_{n}\right)_{n}$ be a sequence of lcs in class $\mathfrak{G}$. Then the topological product $E:=\prod_{n} E_{n}$ belongs to class $\mathfrak{G}$.

(iii) If $E$ is a lcs in class $\mathfrak{G}$ and $F$ is a closed subspace, then the quotient space $E / F$ belongs to class $\mathfrak{G}$.

(iv) Every subspace $F$ of a lcs $E$ in class $\mathfrak{G}$ belongs to class $\mathfrak{G}$.

(v) The completion $F$ of a lcs $E$ in class $\mathfrak{G}$ belongs to class $\mathfrak{G}$.

Next we provide short arguments showing that $(D F)$-spaces and $(L M)$-spaces $E$ admit a $\mathfrak{G}$-representation [11]:

Let $E$ be a $(D F)$-space. Let $\left(B_{n}\right)$ be a fundamental sequence of absolutely convex bounded subsets of a $(D F)$-space $E$. For every $\alpha=\left(n_{k}\right) \in \mathbb{N}^{\mathbb{N}}$ set

$$
A_{\alpha}:=\bigcap_{k} n_{k}\left(B_{k}\right)^{\circ} \text {. }
$$

Clearly conditions (a), (b) and (c) are satisfied. Note also that for a bounded subset $B$ in $\left(E^{\prime}, \beta\left(E^{\prime}, E\right)\right)$ there exists $\alpha \in \mathbb{N}^{\mathbb{N}}$ such that $A_{\alpha}$ absorbs $B$.

Let $E$ be an $(L M)$-space, i.e. the inductive limit of an increasing sequence $\left(E_{n}\right)_{n}$ of metrizable lcs. For every $j \in \mathbb{N}$ let $\left(U_{n}^{j}\right)_{n}$ be a decreasing basis of absolutely convex neighborhoods of zero in $E_{j}$ such that

$$
U_{n+1}^{j}+U_{n+1}^{j} \subset U_{n}^{j}
$$

for all $j, n \in \mathbb{N}$. For every $\alpha=\left(n_{k}\right) \in \mathbb{N}^{\mathbb{N}}$ set

$$
A_{\alpha}:=\bigcap_{k}\left(U_{n_{k}}^{k}\right)^{\circ} .
$$

Clearly conditions (a), (b), (c) are satisfied and each bounded set $B$ in the space $\left(E^{\prime}, \beta\left(E^{\prime}, E\right)\right)$ is equicontinuous. Therefore its polar $D$ is a neighborhood of zero in $E$. Hence there exists a sequence $\alpha=\left(n_{k}\right)$ in $\mathbb{N}^{\mathbb{N}}$ such that

$$
U_{n_{1}}^{1}+U_{n_{2}}^{2}+\cdots+U_{n_{k}}^{k} \subset D
$$

for any $k \in \mathbb{N}$. Consequently

$$
B \subset D^{\circ} \subset \bigcap_{k}\left(U_{n_{k}}^{k}\right)^{\circ}
$$

Spaces in class $\mathfrak{G}$ enjoy another important general property. Theorem 4 below has been proved in [12] by Cascales and Orihuela; we add a simple and short proof due to Ferrando, Kąkol and López-Pellicer from [23].

Theorem 4 Every precompact set in a lcs $E$ in the class $\mathfrak{G}$ is metrizable.

Proof Let $\left\{A_{\alpha}: \alpha \in \mathbb{N}^{\mathbb{N}}\right\}$ be a $\mathfrak{G}$-representation of $E$. For $\alpha=\left(n_{k}\right) \in \mathbb{N}^{\mathbb{N}}$ us set

$$
C_{n_{1}, \ldots, n_{k}}:=\bigcup\left\{A_{\beta}: \beta=\left(m_{k}\right) \in \mathbb{N}^{\mathbb{N}}, n_{j}=m_{j}, 1 \leq j \leq k\right\} .
$$

Let $P$ be a precompact set in $E$. Since the completion of a lcs in class $\mathfrak{G}$ belongs to class $\mathfrak{G}$, we may assume that the precompact set $P$ is compact. Note that for each $\epsilon>0$ there is a countable subset $H_{\epsilon}$ in $E^{\prime}$ such that

$$
E^{\prime}=H_{\epsilon}+\epsilon(P)^{\circ} \text {. }
$$


Indeed, assume this fails. Then (by Zorn's lemma) there exist an uncountable subset $F$ in $E^{\prime}$ and $\epsilon>0$ such that the condition $f-g \in \epsilon(P)^{\circ}$ for $f, g \in F$ implies $f=g$. By an obvious induction procedure we obtain a sequence $\left(n_{k}\right)_{k}$ in $\mathbb{N}$ and a sequence $\left(f_{k}\right)_{k}$ in $E^{\prime}$ of different elements with $f_{k} \in C_{n_{1}, n_{2}, \ldots, n_{k}}$ such that the condition

$$
f_{n}-f_{m} \in \epsilon(P)^{\circ}
$$

implies $m=n$. Indeed, there exists $n_{1} \in \mathbb{N}$ such that $F \cap C_{n_{1}}$ is uncountable. Choose $f_{1} \in F \cap C_{n_{1}}$. Since

$$
C_{n_{1}}=\bigcup\left\{C_{n_{1}, m_{2}}: m_{2} \in \mathbb{N}\right\}
$$

there exists $n_{2} \in \mathbb{N}$ such that $\left(F \backslash\left\{f_{1}\right\}\right) \cap C_{n_{1}, n_{2}}$ is uncountable. Select

$$
f_{2} \in\left(F \backslash\left\{f_{1}\right\}\right) \cap C_{n_{1}, n_{2}} .
$$

Using a simple induction we obtain both sequences as desired. But $f_{k} \in C_{n_{1}, n_{2}, \ldots, n_{k}}$ for all $k \in \mathbb{N}$, so the sequence $\left(f_{k}\right)_{k}$ is equicontinuous. Indeed, for every $k \in \mathbb{N}$ there exists $\beta_{k}=\left(m_{n}^{k}\right)_{n} \in \mathbb{N}^{\mathbb{N}}$ such that $f_{k} \in A_{\beta_{k}}$, where $n_{j}=m_{j}^{k}$ for $j=1,2, \ldots, k$. Define

$$
a_{n}=\max \left\{m_{n}^{k}: k \in \mathbb{N}\right\}
$$

and $\gamma=\left(a_{n}\right) \in \mathbb{N}^{\mathbb{N}}$. Note that $\gamma \geq \beta_{k}$ for every $k \in \mathbb{N}$. Therefore $A_{\beta_{k}} \subset A_{\gamma}$, so $f_{k} \in A_{\gamma}$ for all $k \in \mathbb{N}$ (by condition (b)). Also by (c) the sequence $\left(f_{k}\right)_{k}$ is equicontinuous. By Ascoli theorem for the Banach space $C_{c}(P)$ one gets two different natural numbers $j, k$ such that

$$
f_{j}-f_{k} \in \epsilon(P)^{\circ},
$$

which yields a contradiction. This proves the claim. Since

$$
H:=\left\{H_{n^{-1}}: n \in \mathbb{N}\right\}
$$

is countable, the topology $\tau_{H}$ on $E$ of the pointwise convergence on $H$ restricted to $P$ is Hausdorff and metrizable and coincides with the original topology of $P$. Hence $P$ is metrizable. The proof is completed.

In order to prove Proposition 8 (and then Proposition 9) we need the following result, see [26, 3.1], due to Fremlin (called angelic lemma). First recall that a topological space $X$ is called angelic if every relatively countably compact set $A$ in $X$ is relatively compact and for every $x \in \bar{A}$ there exists a sequence from $A$ converging to $x$.

Theorem 5 Let $X$ and $Y$ be topological spaces where $X$ regular, and let $\Phi: X \rightarrow Y$ be an injective and continuous map. If $A \subset X$ is relatively countably compact and for each $B \subset \Phi(A)$ and $y \in \bar{B}$ there exists a sequence $\left(y_{n}\right)_{n}$ in $B$ converging to $y$, then $\Phi(\bar{A})$ is closed and $\Phi \mid \bar{A}$ is a homeomorphism.

Since precompact sets in a lcs in class $\mathfrak{G}$ are metrizable, then each lcs in class $\mathfrak{G}$ is angelic. The following stronger fact also holds, see [12, Theorem 11].

Proposition 8 Let $E$ be a lcs such that $\left(E^{\prime}, \sigma\left(E^{\prime}, E\right)\right)$ contains a total web-compact subset $X$. Then $\left(E, \sigma\left(E, E^{\prime}\right)\right)$ is angelic and every weakly compact subset of $E$ is metrizable if, and only if, it is contained in a separable subspace of $E$. 
Proof By [52, Theorem 3] the space $C_{p}(X)$ is angelic. Let $A$ be a relatively countably compact subset of $\left(E, \sigma\left(E, E^{\prime}\right)\right)$ and let

$$
\Phi:\left(E, \sigma\left(E, E^{\prime}\right)\right) \rightarrow C_{p}(X)
$$

be a continuous and injective map defined by restriction. Then $\Phi(A)$ is a relatively countably compact subset of $C_{p}(X)$ and then, by the angelicity of $C_{p}(X)$ one gets that $\overline{\Phi(A)}$ is compact and for each subset $B$ of $\Phi(A)$ and each $b \in \bar{B}$ there exists a sequence $\left(b_{n}\right)_{n}$ in $B$ such that $b=\lim _{n} b_{n}$. Then, by Theorem 5 the set $\Phi(\bar{A})$ is closed, because $\Phi(\bar{A})=\overline{\Phi(A)}$ and $\Phi \mid \bar{A}$ is a homeomorphism. Therefore $\bar{A}$ is a compact subset of $\left(E, \sigma\left(E, E^{\prime}\right)\right)$ and for each $a \in \bar{A}$ there exists a sequence $\left(a_{n}\right)$ in $A$ such that $a=\lim _{n} a_{n}$. This proves that $\left(E, \sigma\left(E, E^{\prime}\right)\right)$ is angelic. The rest of the proposition is straightforward.

This yields the following general fact about spaces in class $\mathfrak{G}$.

Proposition 9 The weak topology $\sigma\left(E, E^{\prime}\right)$ of a lcs $E$ in class $\mathfrak{G}$ is angelic.

A classical result of Kaplansky, see [26, Theorem, p. 37] states that if $X$ is a $\sigma$-compact space and $Z$ is a metric space, then $C_{p}(X, Z)$ has countable tightness. This applies to show that the weak topology of a metrizable lcs $E$ has countable tightness, i.e., each adherent point of every subset $A$ is adherent point of a countable subset of $A$. Indeed, since $E$ is metrizable, then $\left(E^{\prime}, \sigma\left(E^{\prime}, E\right)\right)$ is $\sigma$-compact, so $C_{p}\left(\left(E^{\prime}, \sigma\left(E^{\prime}, E\right)\right)\right.$ has countable tightness by Kaplansky Theorem. Since we have $\left(E, \sigma\left(E, E^{\prime}\right)\right) \subset C_{p}\left(\left(E^{\prime}, \sigma\left(E^{\prime}, E\right)\right)\right.$, then $\left(E, \sigma\left(E, E^{\prime}\right)\right)$ has also countable tightness.

Valdivia [71] proved that if $E$ is a Fréchet space and $F$ is its strong dual, then the space $\left(F^{\prime}, \sigma\left(F^{\prime}, F\right)\right)$ is quasi-Suslin, although $\left(F^{\prime}, \sigma\left(F^{\prime}, F\right)\right)$ is $K$-analytic if, and only if, $\left(F, \mu\left(F, F^{\prime}\right)\right)$ is barrelled. Clearly $F$, as a $(D F)$-space, has a closed $\mathfrak{G}$-representation. This result motivates next Theorem 6 which also extends [6, Proposition 1.1], see [25].

We shall say that a subset $A \subset E$ is full if it contains all adherent points in $E$ of sequences from $A$. The following result from [25] provides many lcs $E$ in class $\mathfrak{G}$ whose weak dual $\left(E^{\prime}, \sigma\left(E^{\prime}, E\right)\right)$ is quasi-Suslin but not $K$-analytic. Early isolated examples of this type were presented by Valdivia [71].

Theorem 6 Let $E$ be a lcs in class $\mathfrak{G}$. Then $\left(E^{\prime}, \sigma\left(E^{\prime}, E\right)\right)$ is a quasi-Suslin space.

Proof Let $\left\{A_{\alpha}: \alpha \in \mathbb{N}^{\mathbb{N}}\right\}$ be a $\mathfrak{G}$-representation for $E$. For each $\alpha \in \mathbb{N}^{\mathbb{N}}$ define

$$
B_{\alpha}:=\bigcup\left\{S^{\circ \circ}: S \subset A_{\alpha},|S| \leq \aleph_{0}\right\} .
$$

As countable unions of countable sets are countable, each sequence $\left(u_{n}\right)_{n}$ in $B_{\alpha}$ belongs to a bipolar of a sequence $\left(v_{n}\right)_{n}$ in in $A_{\alpha}$ which is clearly equicontinuous by the assumptions on $\left\{A_{\alpha}: \alpha \in \mathbb{N}^{\mathbb{N}}\right\}$. Using the Alaoglu-Bourbaki and bipolar theorems one gets that the set $\left\{v_{n}: n \in \mathbb{N}\right\}^{\circ \circ}$ is absolutely convex, equicontinuous and $\sigma\left(E^{\prime}, E\right)$-compact. Hence $B_{\alpha}$ is absolutely convex and $\left(u_{n}\right)_{n}$ has cluster points which belong to the set

$$
\left\{v_{n}: n \in \mathbb{N}\right\}^{\circ \circ} \subset B_{\alpha} .
$$

This proves that $B_{\alpha}$ is absolutely convex, weakly countably compact and full. Also the family $\left\{B_{\alpha}: \alpha \in \mathbb{N}^{\mathbb{N}}\right\}$ is a $\mathfrak{G}$-representation of $E$. Now we are ready to apply [6, Proposition 1] to deduce that $E$ is quasi-Suslin.

Theorem 6 can be used to provide a large class of lcs whose weak dual is $K$-analytic. First we recall the following general fact, see [6]. 
Lemma 3 Let $X$ be an angelic space. Then $E$ is quasi-Suslin if, and only if, $X$ is $K$-analytic if, and only if, $X$ admits a compact resolution.

Now we note the following

Corollary 1 Let $E$ be a locally convex space in class $\mathfrak{G}$ such that $\left(E, \sigma\left(E, E^{\prime}\right)\right)$ is webcompact. Then $\left(E^{\prime}, \sigma\left(E^{\prime}, E\right)\right)$ is $K$-analytic. In particular, every separable lcs in class $\mathfrak{G}$ has its precompact dual $\left(E^{\prime}, \tau_{p}\right)$ analytic.

Proof Since $\left(E^{\prime}, \sigma\left(E^{\prime}, E\right)\right)$ is a subspace of $C_{p}\left(E, \sigma\left(E, E^{\prime}\right)\right)$ (the last space is angelic by [52, Theorem 2]) we deduce that $\left(E^{\prime}, \sigma\left(E^{\prime}, E\right)\right)$ is angelic. By Theorem 6 the space $\left(E^{\prime}\right.$, $\left.\sigma\left(E^{\prime}, E\right)\right)$ is quasi-Suslin. Now it is enough to apply Lemma 3 to complete the proof of the first part of this corollary. The rest of the corollary is straightforward (see also Proposition 16).

Being motivated by Kaplansky result mentioned above we characterize those spaces in class $\mathfrak{G}$ which have countable tightness for the weak topology $\sigma\left(E, E^{\prime}\right)$. We shall need the following characterization of weakly realcompact lcs due to Corson, see [71, Page 137].

Proposition 10 Let $\left(E, E^{\prime}\right)$ be a dual pair and let $\left\{F_{i}: i \in I\right\}$ be the family of all separable closed subspaces of $\left(E^{\prime}, \sigma\left(E^{\prime}, E\right)\right)$. Then the following statements are equivalent:

(i) $\left(E, \sigma\left(E, E^{\prime}\right)\right)$ is realcompact;

(ii) $E=\left\{x^{*} \in\left(E^{\prime}\right)^{*}: x^{*} \mid F_{i}\right.$ is $\sigma\left(E^{\prime}, E\right)$-continuous for each $\left.i \in I\right\}$.

We need also the following simple

Lemma 4 Let $E$ be a lcs whose weak topology $\sigma\left(E, E^{\prime}\right)$ has countable tightness. Then $\left(E^{\prime}, \sigma\left(E^{\prime}, E\right)\right)$ is realcompact.

Proof By Corson criterium Proposition 10, it is enough to show that every linear functional $f$ on $E$ which is $\sigma\left(E, E^{\prime}\right)$-continuous on each $\sigma\left(E, E^{\prime}\right)$-closed separable vector subspace is continuous. Observe that the kernel $K:=f^{-1}(0)$ is closed in $E$. In fact, if $y \in \bar{K}$, then there is countable $D \subset K$ with $y \in \bar{D}$ (the closure in $\sigma\left(E, E^{\prime}\right)$ ). By assumption we have $f \mid \overline{\operatorname{lin}(D)}$ is $\sigma\left(E, E^{\prime}\right)$-continuous; hence

$$
f(y) \in \overline{f(\operatorname{lin}(D))} \subset \overline{f(K)}=\{0\},
$$

so $y \in K$ and $f \in E^{\prime}$.

We are ready to prove the following important result from [7].

Theorem 7 Let $E$ be a lcs in the class $\mathfrak{G}$. The following statements are equivalent:

(i) $\left(E, \sigma\left(E, E^{\prime}\right)\right)$ has countable tightness;

(ii) $\left(E^{\prime}, \sigma\left(E^{\prime}, E\right)\right)$ is realcompact.

(iii) $\left(E^{\prime}, \sigma\left(E^{\prime}, E\right)\right)$ is $K$-analytic.

(iv) $\left(E^{\prime}, \sigma\left(E^{\prime}, E\right)\right)^{n}$ is Lindelöf for every $n \in \mathbb{N}$.

(v) $\left(E^{\prime}, \sigma\left(E^{\prime}, E\right)\right)$ is Lindelöf.

Proof (i) $\Rightarrow$ (ii): By Lemma 4 we know that $\left(E^{\prime}, \sigma\left(E^{\prime}, E\right)\right)$ is realcompact.

(ii) $\Rightarrow$ (iii): By Theorem 6 the weak dual $\left(E^{\prime}, \sigma\left(E^{\prime}, E\right)\right)$ is quasi-Suslin. Now Proposition 5 implies that $v\left(E^{\prime}, \sigma\left(E^{\prime}, E\right)\right)=\left(E^{\prime}, \sigma\left(E^{\prime}, E\right)\right)$ is $K$-analytic.

(iii) $\Rightarrow$ (iv): Any countably product of $K$-analytic spaces is $K$-analytic, so it is Lindelöf. 
(iv) $\Rightarrow$ (v): Obvious.

(v) $\Rightarrow$ (i): By Theorem 6 we know that $\left(E^{\prime}, \sigma\left(E^{\prime}, E\right)\right)$ is quasi-Suslin. But every Lindelöf quasi-Suslin space is $K$-analytic. By [2, Theorem I.1.1] the space $C_{p}\left(\left(E^{\prime}, \sigma\left(E^{\prime}, E\right)\right)\right.$ has countable tightness. Therefore since we have $\left(E, \sigma\left(E, E^{\prime}\right)\right) \subset C_{p}\left(\left(E^{\prime}, \sigma\left(E^{\prime}, E\right)\right)\right.$ we note that $\left(E, \sigma\left(E, E^{\prime}\right)\right)$ has countable tightness as well.

On the other hand, [7] we proved

Proposition 11 If $E$ is a a locally convex quasibarrelled space in class $\mathfrak{G}$, then $E$ and $\left(E, \sigma\left(E, E^{\prime}\right)\right)$ have countable tightness.

It turns out that for many spaces in class $\mathfrak{G}$ the converse to Proposition 11 also holds. In [24] we proved among other results the following

Proposition 12 Every (DF)-space with countable tightness is quasibarrelled.

Proof Let $\beta^{*}\left(E, E^{\prime}\right)$ be the topology on $E$ of uniform convergence on $\beta\left(E^{\prime}, E\right)$-bounded sets and let $\xi$ be the original topology of $E$. Clearly $\xi \leq \beta^{*}\left(E, E^{\prime}\right)$. To show that $E$ is quasibarrelled it is enough to show that $\beta^{*}\left(E, E^{\prime}\right)|B=\xi| B$ for each $\xi$-bounded set $B$ in $E$, see [44, 29.3.(2)]. Let $B$ be an absolutely convex $\xi$-closed $\xi$-bounded set. We show that every $\beta^{*}\left(E, E^{\prime}\right)$-closed set $D \subset B$ is $\xi$-closed. Let $x \in \bar{D}$, where the closure is taken in $\xi$. Since by assumption $(E, \xi)$ has countable tightness, then there exists a countable set $C \subset D$ such that $x \in \bar{C}$. Let $C_{0}$ be the closed linear span of $C \cup\{x\}$. Then

$$
\beta^{*}\left(E, E^{\prime}\right)\left|C_{0}=\xi\right| C_{0}
$$

by $[44,29.3(8)]$. Hence $x$ belongs to the $\beta^{*}\left(E, E^{\prime}\right)$-closure of $C$, so $x \in D$.

Remark 1 In [7] we provided examples of $(D F)$-spaces $E$ whose weak topology $\sigma\left(E, E^{\prime}\right)$ does not have countable tightness. By 7 we deduce that $\left(E^{\prime}, \sigma\left(E^{\prime}, E\right)\right)$ is not Lindelöf hence is not a Lindelöf $\Sigma$-space. On the other hand, by Theorem 6 the space $\left(E^{\prime}, \sigma\left(E^{\prime}, E\right)\right)$ is quasi-Suslin.

Proposition 12 applies to provide another characterization for a Fréchet space to be a distinguished space. Recall that a Fréchet space $E$ is called distinguished if the strong dual $\left(E^{\prime}, \beta\left(E^{\prime}, E\right)\right)$ of $E$ is quasibarrelled (equivalently barrelled or bornological), see [5]. Since the strong dual $\left(E^{\prime}, \beta\left(E^{\prime}, E\right)\right)$ of a Fréchet space $E$ is a $(D F)$-space, then Proposition 12 yields the following characterization.

Proposition 13 A Fréchet space $E$ is distinguished if, and only if, $\left(E^{\prime}, \beta\left(E^{\prime}, E\right)\right)$ has countable tightness.

Last Proposition 13 has been used [24] to study non distinguished Köthe echelon spaces. We showed in [35] also the following

Proposition 14 For a $\ell^{\infty}$-barrelled lcs $E$ the following conditions are equivalent:

(i) $\left(E^{\prime}, \sigma\left(E^{\prime}, E\right)\right)$ is a Lindelöf $\Sigma$-space.

(ii) $\left(E, \sigma\left(E, E^{\prime}\right)\right)$ has countable tightness and $E$ is covered by a family $\left\{A_{\alpha}: \alpha=\left(a_{n}\right) \in\right.$ $\Sigma$ \} of sets for some $\Sigma \subset \mathbb{N}^{\mathbb{N}}$ such that each sequence $x_{k} \in C_{n_{1}, n_{n}, \ldots, n_{k}}$ is bounded in $E$, where $C_{n_{1}, n_{n}, \ldots, n_{k}}:=\bigcup\left\{A_{\beta}=\left(b_{n}\right) \in \Sigma: a_{n}=b_{n}, n \leq k\right\}$. 
Proposition 14 at the first glance looks somewhat technical but it covers many concrete classes of topological vector spaces, for example each $(d f)$-space $C_{c}(X)$ has its weak dual Lindelöf $\Sigma$ as $\ell^{\infty}$-barrelled by [38, Corollary 3.3] and having a family of sets as in (ii): If $\left(S_{n}\right)_{n}$ is a fundamental sequence of bounded sets in $C_{c}(X)$ set $A_{\alpha}:=\bigcap_{n} a_{n} S_{n}$ for $\alpha=\left(a_{n}\right) \in \mathbb{N}^{\mathbb{N}}$.

From the definition of the property to be in class $\mathfrak{G}$ it follows that $E^{\prime}$ admits a resolution consisting of $\sigma\left(E^{\prime}, E\right)$-relatively countable compact sets. This implies that $\left(E^{\prime}, \sigma\left(E^{\prime}, E\right)\right)$ is web-compact in sense of Orihuela [52]. But then by [52, Theorem 3] it follows that $C_{p}\left(\left(E^{\prime}, \sigma\left(E^{\prime}, E\right)\right)\right.$ is angelic. Hence

$$
\left(E, \sigma\left(E, E^{\prime}\right)\right) \subset C_{p}\left(\left(E^{\prime}, \sigma\left(E^{\prime}, E\right)\right)\right.
$$

is angelic (this property is a particular case of Proposition 8). This fact covers many of important classes of spaces except spaces $C_{p}(X)$. Indeed, in [8] we proved that $C_{p}(X)$ for uncountable spaces $X$ does not belong to class $\mathfrak{G}$. Nevertheless, it is known that $C_{p}(X, E)$ is weakly angelic for any web-compact $X$ and lcs $E$ in class $\mathfrak{G}$, see [11, Theorem 8, Corollary 1.8]. We provide a direct proof of this fact.

Proposition 15 If $X$ is a web-compact space and $E$ is a lcs in class $\mathfrak{G}$, then $C_{p}(X, E)$ is weakly angelic. If $E \in \mathfrak{G}$ is separable and $C_{p}(X)$ is angelic, then $C_{p}\left(X, E_{\sigma}\right)$ is also angelic, where $E_{\sigma}$ means $\left(E, \sigma\left(E, E^{\prime}\right)\right)$.

Proof Let $\left\{A_{\alpha}: \alpha \in \Sigma\right\}$ be a web-compact representation for $X$. Set $G:=C_{p}(X, E)$. By Proposition 8 it is enough to show that $\left(G^{\prime}, \sigma\left(G^{\prime}, G\right)\right)$ contains a total web-compact subset. If $\left(g_{t}\right)_{t} \rightarrow g$ in $C_{p}(X, E)$, then for each $s \in X$ and each $x^{\prime} \in E^{\prime}$ we have that

$$
\left(x^{\prime} g_{t}(s)\right)_{t} \rightarrow x^{\prime} g(s) .
$$

Therefore the map

$$
\delta_{s x^{\prime}}: C_{p}(X, E) \rightarrow \mathbb{R}
$$

defined by

$$
\delta_{s x^{\prime}}(g):=x^{\prime} g(s)
$$

is continuous. The set

$$
Z:=\left\{\delta_{s x^{\prime}}: s \in \bigcup\left\{A_{\alpha}: \alpha \in \Sigma\right\}, x^{\prime} \in E^{\prime}\right\}
$$

is a total subset of $\left(G^{\prime}, \sigma\left(G^{\prime}, G\right)\right)$. Indeed, if $f \in C_{p}(X, E)$ and

$$
0=\delta_{s x^{\prime}}(f)=x^{\prime} f(s)
$$

for each $s \in \bigcup\left\{A_{\alpha}: \alpha \in \Sigma\right\}$ and $x^{\prime} \in E^{\prime}$, then $f(s)=0$ for each $s \in \bigcup\left\{A_{\alpha}: \alpha \in \Sigma\right\}$. Then by continuity, $f(s)=0$ for each $s \in X$. This implies that $f=0$. Hence $Z$ is a total subset.

Let $E^{\prime}=\bigcup\left\{B_{\beta}: \beta \in \mathbb{N}^{\mathbb{N}}\right\}$ be a $\mathfrak{G}$-representation of $E$. Then

$$
Z=\bigcup\left\{D_{\alpha \beta}:(\alpha, \beta) \in \Sigma \times \mathbb{N}^{\mathbb{N}}\right\},
$$

where

$$
D_{\alpha \beta}=\left\{\delta_{s x^{\prime}}: s \in A_{\alpha}, x^{\prime} \in B_{\beta}\right\}
$$


To prove that the $\sigma\left(G^{\prime}, G\right)$-closure of $Z$ is a web-compact subspace of $\left(G^{\prime}, \sigma\left(G^{\prime}, G\right)\right)$ we need to prove that if $\left(\left(\alpha_{n}, \beta_{n}\right)\right)_{n} \rightarrow(\alpha, \beta)$ in $\Sigma \times \mathbb{N}^{\mathbb{N}}$ and for each $n \in \mathbb{N}$

$$
\delta_{s_{n} x_{n}^{\prime}} \in D_{\alpha_{n} \beta_{n}}
$$

then the sequence $\left(\delta_{s_{n} x_{n}^{\prime}}\right)_{n}$ has an adherent point in $\left(G^{\prime}, \sigma\left(G^{\prime}, G\right)\right)$. As

$$
\left(\left(\alpha_{n}, \beta_{n}\right)\right)_{n} \rightarrow(\alpha, \beta)
$$

we have that $\left\{s_{n}: n \in \mathbb{N}\right\}$ is a relatively countably compact subset of $X$ and $\left\{x_{n}^{\prime}: n \in \mathbb{N}\right\}$ is an equicontinuous subset of $B_{\gamma}$, being $\gamma$ an element of $\mathbb{N}^{\mathbb{N}}$ that verifies that $\beta_{n} \leq \gamma$ for each $n \in \mathbb{N}$. Then the sequence $\left(\delta_{s_{n}} x_{n}^{\prime}\right)_{n}$ has a subnet

$$
\left(\delta_{s_{n(d)} x_{n(d)}^{\prime}}\right)_{d \in D}
$$

such that $\left(s_{n(d)}\right)_{d \in D} \rightarrow s \in X$ and

$$
\left(x_{n(d)}^{\prime}\right)_{d \in D} \rightarrow x^{\prime}
$$

in $\left(E^{\prime}, \sigma\left(E^{\prime}, E\right)\right)$. From equicontinuity it follows that $\left(x_{n(d)}^{\prime}\right)_{d \in D} \rightarrow x^{\prime}$ uniformly on the precompact subsets of $E$. Then the proof will be finished if we prove that

$$
\left(\delta_{s_{n(d)} x_{n(d)}^{\prime}}\right)_{d \in D} \rightarrow \delta_{s x^{\prime}}
$$

in $\left(G^{\prime}, \sigma\left(G^{\prime}, G\right)\right)$. In other words, we have to prove that for each $f \in C(X, E)$ we have that

$$
\lim _{d \in D} x_{n(d)}^{\prime}\left[f\left(s_{n(d)}\right)\right]=x^{\prime}[f(s)] .
$$

But this equality follows from the following facts:

(*) $\lim _{d \in D} x^{\prime}\left[f\left(s_{n(d)}\right)\right]=x^{\prime}[f(s)]$.

(**) As $\left\{f\left(s_{n}\right): n \in \mathbb{N}\right\}$ is a relatively countably compact subset of $E$, and therefore precompact, then

$$
\lim _{d \in D} x_{n(d)}^{\prime}\left[f\left(s_{n(d)}\right)\right]=\lim _{d \in D} x^{\prime}\left[f\left(s_{n(d)}\right)\right] .
$$

Now assume that $E$ is separable. Then $\left(E^{\prime}, \sigma\left(E^{\prime}, E\right)\right)$ is separable by Corollary 1 . If $G$ is a countable and dense subset in $\left(E^{\prime}, \sigma\left(E^{\prime}, E\right)\right)$, then $\xi:=\sigma(E, G)$ is a metrizable locally convex topology on $E$ with $\xi \leq \sigma\left(E, E^{\prime}\right)$. The assumptions of the Fremlin theorem from [26, 3.5] are satisfied: $C_{p}(X)$ is angelic and $E_{\xi}$ is metrizable, so $C_{p}\left(X, E_{\xi}\right)$ is angelic, where $E_{\xi}:=(E, \xi)$. Note that $C_{p}\left(X, E_{\xi}\right) \leq C_{p}\left(X, E_{\sigma}\right)$. Now angelic lemma [26, 3.1; see also Theorem 5] applies to deduce that $C_{p}\left(X, E_{\sigma}\right)$ is angelic.

Applying last Proposition 15 we provide the following extension of main result of [68].

Theorem 8 Let $E$ be a separable lcs in class $\mathfrak{G}$ and let $\xi$ be a topology on $C(X, E)$ stronger than the pointwise topology of $C(X, E)$. The following assertions are equivalent:

(i) $(C(X, E), \xi)$ is $K$-analytic.

(ii) $(C(X, E), \xi)$ admits a compact resolution.

(iii) $(C(X, E), \xi)$ admits a relatively countably compact resolution.

Proof Since each $K$-analytic space admits a compact resolution, it is enough to show that

(iii) $\Rightarrow$ (i): If $(C(X, E), \xi)$ admits a relatively countably compact resolution $\left\{K_{\alpha}: \alpha \in\right.$ $\left.\mathbb{N}^{\mathbb{N}}\right\}$, then $\left\{K_{\alpha}: \alpha \in \mathbb{N}^{\mathbb{N}}\right\}$ is a bounded resolution in $C_{p}(X, E)$ in the pointwise topology $\tau_{p}$. Since $C_{p}(X)$ is isomorphic with a subspace of $C_{p}(X, E)$, then $C_{p}(X)$ admits a bounded 
resolution. By Proposition 6 the space $C_{p}(X)$ is angelic. Now we apply Proposition 15 to get that $C_{p}\left(X, E_{\sigma}\right)$ is angelic. Hence the space $C_{p}(X, E)$ is angelic and then $(C(X, E), \xi)$ is also angelic (see Theorem 5). Finally note that by Lemma 3 the space $(C(X, E), \xi)$ is $K$-analytic.

Lemma 3 implies that if a topological space $(X, \xi)$ admits a weaker metric topology, then $(X, \xi)$ is $K$-analytic iff $(X, \xi)$ has a compact resolution. It turns out that this situation implies that $(X, \xi)$ is even analytic, i.e. $(X, \xi)$ is a continuous image of the space $\mathbb{N}^{\mathbb{N}}$. The following applicable result was obtained by Talagrand; the proof presented below is a modification of the proof due to Cascales and Oncina, see [10, Corollary 4.3], see also [59, Theorem 5.5.1] and [61, Corollary 1, p.105].

Proposition 16 Let $(X, \tau)$ be a $K$-analytic space and let d be a metric on $X$ whose topology is coarser than $\tau$. Then $(X, \tau)$ is analytic. Every regular analytic space $X$ admits a weaker metric topology.

Proof Let $\left\{K_{\alpha}: \alpha \in \mathbb{N}^{\mathbb{N}}\right\}$ be a compact resolution on $(X, \tau)$ and $\left\{z_{n}: n \in \mathbb{N}\right\}$ be a dense subset of $(X, d)$. By $B_{d}(z, r)$ denote the $d$-closed ball in $(X, d)$ of center $z$ and radius $r>0$. For $\beta=\left(b_{n}\right) \in \mathbb{N}^{\mathbb{N}}$ let

$$
D_{\beta}:=\bigcap_{n \in \mathbb{N}} B_{d}\left(z_{b_{n}}, n^{-1}\right)
$$

Each set $D_{\beta}$ is unitary or void. For $y \in X$ there exists $(\alpha, \beta) \in \mathbb{N}^{\mathbb{N}} \times \mathbb{N}^{\mathbb{N}}$ such that $K_{\alpha} \cap D_{\beta}=\{y\}$.

For $K_{\alpha} \cap D_{\beta} \neq \emptyset$, we denote by $y_{\alpha \beta}$ the element of $X$ such that $K_{\alpha} \cap D_{\beta}=\left\{y_{\alpha \beta}\right\}$. If

$$
T:=\left\{(\alpha, \beta) \in \mathbb{N}^{\mathbb{N}} \times \mathbb{N}^{\mathbb{N}}: \emptyset \neq K_{\alpha} \cap D_{\beta}=\left\{y_{\alpha \beta}\right\}\right\},
$$

then the map $f: T \rightarrow X$ defined by $f((\alpha, \beta))=y_{\alpha \beta}$ is onto.

Let $(\alpha(p), \beta(p))_{p}$ be a sequence in $T$ that converges to $(\alpha, \beta)$ in $\mathbb{N}^{\mathbb{N}} \times \mathbb{N}^{\mathbb{N}}$ and let $(\alpha(p), \beta(p))_{p(m)}$ be a subsequence.

By $K$-analyticity we deduce that $\left(y_{\alpha(p), \beta(p)}\right)_{p(m)}$ has an adherent point $y \in K_{\alpha}$. Since $\beta(p)$ converges to $\beta=\left(b_{n}\right)_{n} \in \mathbb{N}^{\mathbb{N}}$, the sequence $\left(y_{\alpha(p), \beta(p)}\right)_{p(m)}$ is eventually in each $B_{d}\left(z_{b_{n}}, n^{-1}\right)$, hence its adherent point $y$ belongs to $B_{d}\left(z_{b_{n}}, n^{-1}\right)$.

This shows that

$$
y \in K_{\alpha} \cap D_{\beta}=\left\{y_{\alpha \beta}\right\} .
$$

We proved that $(\alpha, \beta) \in T$, i.e. $T$ is a closed subset of $\mathbb{N}^{\mathbb{N}} \times \mathbb{N}^{\mathbb{N}}$ and, therefore, $T$ is a Polish space. Moreover we proved that $y_{\alpha \beta}$ is an adherent point of each subsequence of $\left(y_{\alpha(p), \beta(p)}\right)_{p}$. This implies trivially that $y_{\alpha(p), \beta(p)}$ converges to $y_{\alpha \beta}$, i.e., $f(\alpha(p), \beta(p))$ converges to $f(\alpha, \beta)$. Therefore $f$ is a continuous mapping from the Polish space $T$ onto $(Y, \tau)$. This proves that $(Y, \tau)$ is analytic.

In order to prove the second part assume that $\Delta=\{(x, x): x \in X\}$ is the diagonal of the analytic space $X \times X$. Clearly $\Delta$ and $(X \times X) \backslash \Delta$ are analytic and, therefore, they are Lindelöf.

If $x \neq y$ there exist two closed neighbourhoods $F_{x}$ and $F_{y}$ of $x$ and $y$, respectively, such that

$$
F_{x} \times F_{y} \subset(X \times X) \backslash \Delta .
$$


The Lindelöf property enables us to determine a sequence $\left(x_{n}, y_{n}\right)_{n}$ such that

$$
X \times X \backslash \Delta=\bigcup_{n} F_{x_{n}} \times F_{y_{n}} .
$$

Therefore $\Delta$ is a $G_{\delta}$-subset of $X \times X$ since $\Delta=\bigcap_{n} G_{n}$, where

$$
G_{n}=(X \times X) \backslash\left(F_{x_{n}} \times F_{y_{n}}\right) .
$$

For each $(x, x) \in \Delta$ and $n \in \mathbb{N}$ there exists an open set $U_{x, n}$ in $X$ such that

$$
(x, x) \in U_{x, n} \times U_{x, n} \subset G_{n}
$$

As the space $X$ is completely regular we may suppose that there exists a continuous function $f_{x, n}: X \rightarrow[0,1]$ such that

$$
\left.\left.f_{x, n}\left(U_{x, n}\right) \subset\right] \frac{1}{2}, 1\right], \quad f_{x, n}\left(X \backslash U_{x, n}\right) \subset\left[0, \frac{1}{2}\right] .
$$

By Lindelöf property of $\Delta$ the family $\left\{U_{x, n}: x \in X\right\}$ contains a sequence $\left(U_{x_{(i, n)}, n}\right)_{i}$ such that

$$
\Delta \subset \bigcup_{i} U_{x_{(i, n)}, n} \times U_{x_{(i, n)}, n}:=G_{n}^{*}
$$

From $\Delta=\bigcap_{n} G_{n}^{*}$ it follows that if $x \neq y$ are two different points of $X$, then there exists $n \in \mathbb{N}$ such that $(x, y) \notin G_{n}^{*}$. Then from $(x, x) \in G_{n}^{*}$ it follows that there exists $j \in \mathbb{N}$ such that $x \in U_{x_{(j, n)}, n}$. This implies that $y \notin U_{x_{(j, n)}, n}$, since $(x, y) \notin G_{n}^{*}$. By construction

$$
f_{x_{(j, n)}, n}(x) \neq f_{x_{(j, n)}, n}(y) .
$$

Then $X$ endowed with the topology that makes continuous the countable family of functions $\left\{f_{x_{(i, n)}, n}:(i, n) \in \mathbb{N}^{2}\right\}$ is metrizable with the metric defined by the formula

$$
d(x, y)=\sum\left\{2^{-i-n}\left|f_{x_{(i, n)}, n}(x)-f_{x_{(i, n)}, n}(y)\right|:(i, n) \in \mathbb{N}^{2}\right\}
$$

Clearly $d(x, y)$ defines a metric topology weaker then $\tau$.

\section{Weakly Lindelöf Fréchet locally convex spaces}

We know already that the weak dual of a quasibarrelled lcs in class $\mathfrak{G}$ is $K$-analytic by Theorem 7 and Proposition 11. In particular this yields that every reflexive Fréchet space is weakly $K$-analytic. Another large class of lcs for which the weak topology is Lindelöf is the class of (WCG) Fréchet locally convex spaces.

In [42] Khurana proved that every (WCG) Fréchet space is weakly $K$-analytic. For the case when $E$ is a (WCG) Banach space we refer to [65], see also [17,53].

Recall that a Banach space $E$ is weakly compactly generated (WCG) if there exists a weakly compact subset in $E$ whose linear span is a dense subspace of $E$. A lcs $E$ is said to be (WCG) if there exists an increasing sequence of $\sigma\left(E, E^{\prime}\right)$-compact subsets of $E$ whose union is dense in $E$.

In [53] Orihuela used the method of constructing projections in (WCG) Banach spaces developed by Valdivia from [72-76] and by Orihuela and Valdivia in [55], to provide a direct proof showing that the weak topology of a (WCG) Banach space is Lindelöf. This method applies to prove [53] that a dual Banach space is weakly Lindelöf if, and only if, its *-weak 
dual unit ball is a Corson compact space. Hence if $E$ is a dual Banach space which is weakly Lindelöf, then the product $E \times E$ is weakly Lindelöf, too.

We refer the reader to $[1,9,54]$ (and references) concerning weakly countably determined (WCD) and weakly Lindelöf determined (WLD) Banach spaces which provided larger classes (of weakly Lindelöf spaces) than the class of (WCG) Banach spaces.

We recall the following theorem due to Khurana [42].

Theorem 9 Let $E$ be a Fréchet space which admits an increasing sequence of $\sigma\left(E, E^{\prime}\right)$ compact sets whose union is dense in $E$. Then $\sigma\left(E, E^{\prime}\right)$ is $K$-analytic. Moreover $E$ is a Borel subset of $\left(E^{\prime \prime}, \sigma\left(E^{\prime \prime}, E^{\prime}\right)\right)$, where $E^{\prime \prime}$ is the bidual of $E$.

Proof Since every metrizable lcs $E$ is angelic in the weak topology $\sigma\left(E, E^{\prime}\right)$, then to prove that $\left(E, \sigma\left(E, E^{\prime}\right)\right)$ is a $K$-analytic space it is enough to show that $\left(E, \sigma\left(E, E^{\prime}\right)\right)$ has a compact resolution by Lemma 3.

In a natural way, we identify $\left(E, \sigma\left(E, E^{\prime}\right)\right)$ with a subspace of $\mathbb{R}^{E^{\prime}}$, endowed with the product topology. Therefore for each $x$ in $E$ we have $x=(g(x))_{g \in E^{\prime}}$. For each $f \in E^{\prime}$ the mapping

$$
P_{f}: \mathbb{R}^{E^{\prime}} \longrightarrow \mathbb{R}
$$

defined by

$$
P_{f}\left(\left(\alpha_{g}\right)_{g \in E^{\prime}}\right):=\alpha_{f}
$$

verifies in any point $x=(g(x))_{g \in E^{\prime}} \in E$

$$
P_{f}(x)=P_{f}\left((g(x))_{g \in E^{\prime}}\right)=f(x)
$$

and therefore, the restriction of $P_{f}$ to $E$ is $f$.

Let $\left(V_{n}\right)_{n}$ be a base of closed absolutely convex neighbourhoods of 0 in $E$, such that

$$
(n+1) V_{n+1} \subset V_{n}
$$

for each $n \in \mathbb{N}$. Let $\bar{V}_{n}$ be the closure of $V_{n}$ in $\mathbb{R}^{E^{\prime}}$. Then, given $n$ and $p$ in $\mathbb{N}, f \in V_{n}^{0}$ and $z_{n+p} \in \bar{V}_{n+p}$, we have that

$$
\left|P_{f}\left(z_{n+p}\right)\right| \leq \sup \left\{\left|P_{f}(x)\right|: x \in \bar{V}_{n+p}\right\}=\sup \left\{\left|P_{f}(x)\right|: x \in V_{n+p}\right\}
$$

From this it follows that for each $f \in V_{n}^{0}$ and $z_{n+p} \in \bar{V}_{n+p}$ we have

$$
\left|P_{f}\left(z_{n+p}\right)\right| \leq \sup \left\{|f(x)|: x \in \frac{V_{n}}{n+p}\right\} \leq \frac{1}{n+p}
$$

Let $\left(A_{n}\right)_{n}$ be an increasing sequence of weakly compact absolutely convex subsets of $E$ such that $\bigcup_{n} A_{n}=H$ is dense in $E$. Since $H$ is dense in $E$ and $V_{n}$ is a neighbourhood of zero in $E$ we have that

$$
E \subset H+V_{n} \subset H+\bar{V}_{n}
$$

for each $n \in \mathbb{N}$. If

$$
x \in \bigcap\left\{H+\bar{V}_{n}: n \in \mathbb{N}\right\}
$$

then there exists a sequence

$$
\left(x=h_{n}+z_{n}\right)_{n}
$$


with $h_{n} \in H$ and $z_{n} \in \bar{V}_{n}$. Fix an $n \in \mathbb{N}$. Then for each $f \in V_{n}^{0}$ and each $p$ and $q$ in $\mathbb{N}$ we have by $P_{f}$ definition and (2) that

$$
\left|f\left(h_{n+p}-h_{n+q}\right)\right|=\left|P_{f}\left(x-z_{n+p}\right)-P_{f}\left(x-z_{n+q}\right)\right|=\left|P_{f}\left(z_{n+q}\right)-P_{f}\left(z_{n+p}\right)\right|
$$

and therefore from (1) we deduce that

$$
\left|f\left(h_{n+p}-h_{n+q}\right)\right| \leq\left|P_{f}\left(z_{n+p}\right)\right|+\left|P_{f}\left(z_{n+q}\right)\right| \leq \frac{2}{n} .
$$

for each $f \in V_{n}^{0}$. The uniformity implies that the sequence $\left(h_{s}\right)_{s}$ is Cauchy in the Fréchet space $E$ and therefore it has a limit $h \in E$. Then $\lim _{s \rightarrow \infty} h_{s}=h$ and from (1) it follows that

$$
P_{f}(x)=\lim _{s \rightarrow \infty} P_{f}\left(h_{s}+z_{s}\right)=\lim _{s \rightarrow \infty}\left\{f\left(h_{s}\right)+P_{f}\left(z_{s}\right)\right\}=f(h)=P_{f}(h)
$$

implying $x=h$ in $\mathbb{R}^{E^{\prime}}$. Since $x=h \in E$, then

$$
E=\bigcap\left\{H+\bar{V}_{n}: n \in \mathbb{N}\right\}
$$

and then we note that

$$
E=\bigcap\left\{\bigcup\left[A_{m}+\overline{V_{n}}: m \in \mathbb{N}\right]: n \in \mathbb{N}\right\} .
$$

Therefore, $E$ admits a resolution $\left\{K_{\alpha}: \alpha \in \mathbb{N}^{\mathbb{N}}\right\}$ with

$$
K_{\alpha}=\bigcap\left\{A_{m_{n}}+\bar{V}_{n}: n \in \mathbb{N}\right\}
$$

for each $\alpha=\left(m_{n}\right) \in \mathbb{N}^{\mathbb{N}}$.

In $\mathbb{R}^{E^{\prime}}$ the sets $A_{m_{n}}+\bar{V}_{n}$ for $n \in \mathbb{N}$, are closed.

Claim. The closed set $K_{\alpha}$ is bounded in $\mathbb{R}^{E^{\prime}}$.

Indeed, if $f \in E^{\prime}$, then there exists $n$ in $\mathbb{N}$ such that $f \in V_{n}^{0}$ and we have

$$
\begin{aligned}
\sup \left\{|f(x)|: x \in K_{\alpha}\right\} & \leq \sup \left\{\left|P_{f}(x)\right|: x \in A_{m_{n}}+\bar{V}_{n}\right\} \\
& \leq \sup \left\{|f(x)|: x \in A_{m_{n}}\right\}+\sup \left\{\left|P_{f}(x)\right|: x \in \bar{V}_{n}\right\} \\
& =\sup \left\{|f(x)|: x \in A_{m_{n}}\right\}+\sup \left\{\left|P_{f}(x)\right|: x \in V_{n}\right\} \\
& \leq k_{m_{n}}+1,
\end{aligned}
$$

where

$$
k_{m_{n}}:=\sup \left\{|f(x)|: x \in A_{m_{n}}\right\} .
$$

This proves that the closed set $K_{\alpha}$ is compact in $\mathbb{R}^{E^{\prime}}$ and it is also compact in $\left(E, \sigma\left(E, E^{\prime}\right)\right)$. We proved that $\left\{K_{\alpha}: \alpha \in \mathbb{N}\right\}$ is a compact resolution in $\left(E, \sigma\left(E, E^{\prime}\right)\right)$.

Finally, since $A_{m_{n}}+\bar{V}_{n}$ is closed in $\mathbb{R}^{E^{\prime}}$, then

$$
\left(A_{m_{n}}+\bar{V}_{n}\right) \bigcap E^{\prime \prime}
$$

is closed in $\left(E^{\prime \prime}, \sigma\left(E^{\prime \prime}, E^{\prime}\right)\right)$ and so

$$
\left(H+\bar{V}_{n}\right) \cap E^{\prime \prime}
$$

is a Borel set in $\left(E^{\prime \prime}, \sigma\left(E^{\prime \prime}, E^{\prime}\right)\right.$. Since

$$
E=\bigcap\left\{\left(H+\bar{V}_{n}\right) \cap E^{\prime \prime}: n \in \mathbb{N}\right\}
$$

we deduce that $E$ is a Borel set in $\left(E^{\prime \prime}, \sigma\left(E^{\prime \prime}, E^{\prime}\right)\right)$. 
It is known [71] that (*) every lcs which is a Baire space and additionally $K$-analytic must be a Fréchet space, i.e. a metrizable and complete lcs. This combined with Theorem 9 yields the following

Proposition 17 Let $E$ be a (WCG) Baire lcs. Then $E$ is a Fréchet space if, and only if, $\left(E, \sigma\left(E, E^{\prime}\right)\right)$ is $K$-analytic.

Proof Assume that $E$ is a Fréchet space. By Theorem 9 the space $E$ is weakly $K$-analytic. Conversely, if $\left(E, \sigma\left(E, E^{\prime}\right)\right)$ is $K$-analytic, then $\left(E, \sigma\left(E, E^{\prime}\right)\right)$ admits a compact resolution. By (*) the space $E$ is metrizable. Since $E$ has a $\sigma\left(E, E^{\prime}\right)$-compact resolution and the original topology of $E$ has a basis of neighbourhoods consisting of $\sigma\left(E, E^{\prime}\right)$-closed sets, then the space $E$ admits a complete resolution as well. But every metrizable Baire lcs which has a complete resolution is complete, see [34, Theorem] and its refined version in [15, Theorem 3.5 and Corollary 3.6].

Proposition 17 applies also to get the well-known fact stating that a separable space $X:=\mathbb{R}^{A}$ with uncountable $A$ is not $K$-analytic (since $X$ is (WCG) and Baire but not metrizable).

If $E$ is a Banach space, then the Mackey dual $\left(E^{\prime}, \mu\left(E^{\prime}, E\right)\right)$ is not metrizable, except the case when $E$ is reflexive. It is well-known that $\left(E^{\prime}, \mu\left(E^{\prime}, E\right)\right)$ is a complete lcs. If $B^{\prime}$ is the dual unit ball in the dual $E^{\prime}$ of $E$, then one may expect that some cases (different from reflexivity in general case) may provide metrizability of $\left(B^{\prime}, \mu\left(E^{\prime}, E\right) \mid B^{\prime}\right)$.

In [62] Schlüchtermann and Wheeller introduced strongly weakly compactly generated ((SWCG) shortly) Banach spaces. A Banach space is (SWCG) if the space $\left(B^{\prime}, \mu\left(E^{\prime}, E\right) \mid B^{\prime}\right)$ is metrizable, see also [63].

The following Theorem 10 (from [62, Theorem 2.1]) characterizes (SWCG) Banach spaces in term of some density condition. Theorem 10 shows also that every (SWCG) Banach space is (WCG).

In [62, Theorem 2.5] it is proved that every (SWCG) Banach space is weakly sequentially complete. Hence the space $c_{0}$ although is a (WCG) space is not (SWCG).

Theorem 10 The following conditions are equivalent for a Banach space $E$ with a closed unit balls $B \subset E$ and $B^{\prime} \subset E^{\prime}$.

(i) $\left(B^{\prime}, \mu\left(E^{\prime}, E\right) \mid B^{\prime}\right)$ is metrizable.

(ii) There exists a sequence $\left(K_{n}\right)_{n}$ of weakly compact absolutely convex subsets of E such that for every weakly compact set $L \subset E$ and every $\epsilon>0$ there exists $n \in \mathbb{N}$ such that $L \subset K_{n}+\epsilon B$.

(iii) There exists a weakly compact absolutely convex set $K \subset E$ such that for each weakly compact set $L \subset E$ and every $\epsilon>0$ there is $n \in \mathbb{N}$ such that $L \subset n K+\epsilon B$.

Assume now that the space $E$ is a separable (SWCG) Banach space. Then clearly the space $\left(E^{\prime}, \mu\left(E^{\prime}, E\right)\right)$ is separable. Since $\left(E^{\prime}, \mu\left(E^{\prime}, E\right)\right)$ is separable, then $B^{\prime}$ is separable as well.

Indeed, let $\mathfrak{F}\left(E^{\prime}\right)$ be the set of all absolutely convex neighbourhoods of zero in $\mu\left(E^{\prime}, E\right)$ and let $U_{m} \in \mathfrak{F}\left(E^{\prime}\right), m \in \mathbb{N}$, such that $\left(B^{\prime} \cap\left[U_{m}+U_{m}\right]\right)_{m}$ is a basis of neighbourhoods of zero in $B^{\prime}$. By separability there exists a countable set $B_{m}$ such that

$$
E^{\prime} \subset B_{m}+U_{m}
$$

and then there exists in $B^{\prime}$ a countable subset $C_{m}$ such that

$$
B^{\prime} \subset C_{m}+U_{m}+U_{m} .
$$


Since $E^{\prime}=\bigcup_{n} n B^{\prime}$ and each $n B^{\prime}$ is metrizable separable and complete, then the space $\left(E^{\prime}, \mu\left(E^{\prime}, E\right)\right)$ is analytic. Therefore we have

Proposition 18 Let $E$ be a $(S W C G)$ Banach space. Then $\left(E^{\prime}, \mu\left(E^{\prime}, E\right)\right)$ is analytic if, and only if, E is separable.

Let $(S, \Sigma, \mu)$ is a finite measure space. $L_{1}(\mu, E)$ denotes a Banach space of Bochner integrable functions on $S$ into a Banach space $E$. In [62, Theorem 3.2] Schlüchtermann and Wheller presented partial results of whether $X$ (SWCG) implies that $L_{1}(\mu, E)$ is Talagrand [67], see also Diestel [13], proved that $L_{1}(\mu, E)$ is (WCG) if $E$ is a (WCG) Banach space.

If $E$ is a separable Banach space, then the Mackey dual $\left(E^{\prime}, \mu\left(E^{\prime}, E\right)\right)$ is a separable but the strong dual $\left(E^{\prime}, \beta\left(E^{\prime}, E\right)\right)$ need not be separable. Clearly $\left(E^{\prime}, \beta\left(E^{\prime}, E\right)\right)$ is analytic if, and only if, $\left(E^{\prime}, \beta\left(E^{\prime}, E\right)\right)$ is separable. Theorem 10 and Proposition 18 may suggest the following question:

Let $E$ be a separable Banach space. Is it true that the Mackey dual $\left(E^{\prime}, \mu\left(E^{\prime}, E\right)\right)$ of $E$ is an analytic space?

For the Mackey dual of $C_{p}(X)$ we proved in [36] the following general fact suggested by [18], where Ferrando proved that the Mackey dual of $C_{p}[0,1]$ is not analytic but weakly analytic.

Theorem 11 The Mackey dual of $C_{p}(X)$ is analytic if, and only if, $X$ is countable.

Proof Set $X:=(X, \tau)$ and assume that the Mackey dual of $C_{p}(X)$ is analytic. Suppose, by contradiction, that $X$ is uncountable. For $x \in X$ the functional

$$
\delta_{x}: C_{p}(X) \longrightarrow \mathbb{R}
$$

defined by $\delta_{x}(f)=f(x)$ is linear and continuous. Denote by $L_{p}(X)$ and $L_{\mu}(X)$ the dual of $C_{p}(X)$ with the weak dual topology $\sigma=\sigma\left(C_{p}(X)^{\prime}, C_{p}(X)\right)$ and with the Mackey topology $\mu=\mu\left(C_{p}(X)^{\prime}, C_{p}(X)\right)$, respectively. Set $Y=\left\{\delta_{x}: x \in X\right\}$. The map

$$
\delta:(X, \tau) \longrightarrow(Y, \sigma \mid Y)
$$

defined by $x \rightarrow \delta_{x}$ is a homeomorphism and the set $Y$ is closed in $L_{p}(X)$, see [2, Proposition 0.5.9]. Hence $Y$ is also closed in $L_{\mu}(X)$. Thus $(Y, \mu \mid Y)$ is analytic. Let $\gamma$ be the topology on $X$ such that $\delta$ is a homeomorphism between $(X, \gamma)$ and $(Y, \mu \mid Y)$. Since $(X, \gamma)$ is an uncountable analytic space, it contains a set $A$ homeomorphic to the Cantor set, see [59]. Clearly

$$
\gamma|A=\tau| A .
$$

Let $\left(x_{n}\right)_{n} \subset A$ be a sequence such that $x_{n} \neq x_{m}$ for $n \neq m$ that converges to some

$$
x_{0} \in\left(A \backslash\left\{x_{n}: n \in \mathbb{N}\right\}\right) .
$$

It is easy to see that for every closed subspace $G$ of $(X, \tau)$ and every $x \in(X \backslash G)$ there exists $f \in C(X, I)$ with $f(x)=1$ such that $G \cap \operatorname{supp} f=\emptyset$. Put

$$
X_{n}=\left\{x_{k}: k>n\right\} \cup\left\{x_{0}\right\}
$$

for $n \in \mathbb{N}$. Clearly $X_{n}$ is closed in $X$ and $x_{n} \notin X_{n}$ for $n \in \mathbb{N}$. Therefore we can construct inductively a sequence $\left(f_{n}\right)_{n} \subset C(X, I)$, such that $f_{n}\left(x_{n}\right)=1$ and

$$
\operatorname{supp} f_{n} \cap\left(X_{n} \cup \bigcup\left\{\operatorname{supp} f_{k}: 1 \leq k<n\right\}\right)=\emptyset \text {. }
$$


Then $\left.x_{0} \notin \bigcup\left\{\operatorname{supp} f_{k}: k \in \mathbb{N}\right\}\right)$ and

$$
\operatorname{supp} f_{n} \cap \operatorname{supp} f_{m}=\varnothing
$$

for all $n, m \in \mathbb{N}$ with $n \neq m$.

Denote by $C^{*}(X)$ the Banach space of all bounded real-valued continuous functions on $X$ with the sup norm $\|\cdot\|$. Let $g \in C^{*}(X)^{\prime}$. For $k \in \mathbb{N}$ we put

$$
\alpha_{k}=\left|g\left(f_{k}\right)\right| / g\left(f_{k}\right)
$$

if $g\left(f_{k}\right) \neq 0$, and $\alpha_{k}=1$, otherwise. Then $\left|\alpha_{k}\right|=1$ and

$$
\alpha_{k} g\left(f_{k}\right)=\left|g\left(f_{k}\right)\right|
$$

for $k \in \mathbb{N}$.

Let $n \in \mathbb{N}$ and $S_{n}=\sum_{k=1}^{n} \alpha_{k} f_{k}$. Then $S_{n} \in C^{*}(X)$ and $\left\|S_{n}\right\|=1$. Thus

$$
\sum_{k=1}^{n}\left|g\left(f_{k}\right)\right|=\left|\sum_{k=1}^{n} \alpha_{k} g\left(f_{k}\right)\right|=\left|g\left(S_{n}\right)\right| \leq\|g\|
$$

for $n \in \mathbb{N}$, so

$$
\sum_{k=1}^{\infty}\left|g\left(f_{k}\right)\right| \leq\|g\| .
$$

Hence $g\left(f_{k}\right) \rightarrow 0$. It follows that the sequence $\left(f_{n}\right)_{n}$ converges weakly to 0 in $C^{*}(X)$. Thus the set

$$
F_{0}=\left\{0, f_{1},-f_{1}, f_{2},-f_{2}, \ldots\right\}
$$

is weakly compact in $C^{*}(X)$. By the Krein-Smulian Weak Compactness Theorem [47, Theorem 2.8.14] the closed convex hull $F$ of $F_{0}$ in $C^{*}(X)$ is weakly compact. Clearly $F$ is the closed absolutely convex hull of the set $\left\{f_{k}: k \in \mathbb{N}\right\}$ in $C^{*}(X)$. The topology $\varrho$ of the pointwise convergence in $C^{*}(X)$ is weaker than the weak topology of $C^{*}(X)$, so $F$ is compact in $\left(C^{*}(X), \varrho\right)$. Hence $F$ is compact in $C_{p}(X)$, since the injection map

$$
\left(C^{*}(X), \varrho\right) \longrightarrow C_{p}(X)
$$

is continuous. Thus the functional

$$
p_{F}: L_{\mu}(X) \longrightarrow[0, \infty)
$$

defined by

$$
p_{F}(g)=\sup \{|g(f)|: f \in F\},
$$

is a continuous seminorm. Since $\left(f_{n}\right)_{n} \subset F$ we have

$$
p_{F}\left(\delta_{x_{n}}\right) \geq\left|f_{n}\left(x_{n}\right)\right|=1
$$

for $n \in \mathbb{N}$. It is easy to see that $f\left(x_{0}\right)=0$ for all $f \in F$, so $p_{F}\left(\delta_{x_{0}}\right)=0$. It follows that $\delta_{x_{n}} \nrightarrow \delta_{x_{0}}$ in $(Y, \mu \mid Y)$, so $x_{n} \not \rightarrow x_{0}$ in $(X, \gamma)$; a contradiction.

Assume now that the space $X$ is countable. If $C_{p}(X)$ is finite-dimensional, then the Mackey dual $L_{\mu}(X)$ of $C_{p}(X)$ is finite-dimensional; so it is analytic. If $C_{p}(X)$ is infinite-dimensional, then $C_{p}(X)$ is a metrizable lcs isomorphic to a dense subspace of $\mathbb{R}^{\mathbb{N}}$, so $L_{\mu}(X)$ is algebraically isomorphic to $\varphi$, the strong dual of $\mathbb{R}^{\mathbb{N}}$. But $\varphi$ with the strongest locally convex topology is the sum of an increasing sequence of finite-dimensional Banach spaces, so it is an analytic space. It follows that $L_{\mu}(X)$ is analytic, too. 
Theorem 11 and its proof yields also the following

Corollary 2 The strong dual of $C_{p}(X)$ is analytic if, and only if, $X$ is countable.

Recall that $L_{p}(X)$ is analytic if, and only if, $X$ is analytic by [2, Proposition 0.4.13]. Thus Theorem 11 provides many of concrete non analytic lcs whose weak topology is analytic.

Corollary 3 Let $X$ be an uncountable analytic space. Then the Mackey dual $L_{\mu}(X)$ of $C_{p}(X)$ is weakly analytic but not analytic.

The Mackey dual $\left(E^{\prime}, \mu\left(E^{\prime}, E\right)\right)$ of a Banach space has been studied also in $[43,63]$. In [63] the authors proved among the others that if $E$ is a separable (SWCG) Banach space, then $\left(E, \sigma\left(E, E^{\prime}\right)\right)$ (which is clearly analytic) is an $\aleph_{0}$-space, i.e., it has a countable pseudobase.

A collection $\mathcal{P}$ of subsets of a topological space $E$ is called a pseudobase if for any open set $U \subset E$ and compact $K \subset U$ there exists $P \in \mathcal{P}$ with $K \subset P \subset U$. Recall also that every $\aleph_{0}$-space is separable and Lindelöf and every closed set is a $G_{\delta}$-set, [48], [63, Theorem 4.1]. In [43] Kirk studied the Mackey dual for spaces $C(K)$ with compact $K$.

On the other hand, by Batt and Hiermeyer [3, 2.6] (see also [62], [63, p. 274] and [63, Theorem 4.2]) there exists a separable Banach space $E$ for which $\left(E, \sigma\left(E, E^{\prime}\right)\right)$ is not an $\aleph_{0}$-space. It is known also [48], [63, Theorem 4.1], that a regular topological space is both an $\aleph_{0}$-space and a $k$-space if, and only if, it is a quotient of a separable metric space. Therefore it seems to be natural to ask when for a Banach space $E$ the space $\left(E, \sigma\left(E, E^{\prime}\right)\right)$ is a $k$-space.

Recall that a Hausdorff space $X$ is a $k$-space if a set $A \subset X$ is closed in $X$ if, and only if, $A \cap K$ is closed in $K$ for each compact set $K \subset X$. We shall need the following fact due to Grothendieck [29, p. 134].

Lemma 5 Let $A \subset E^{\prime}$ be $\mu\left(E^{\prime}, E\right)$-compact for a Banach space $E$. Then every $\sigma\left(E, E^{\prime}\right)$ convergent sequence in $E$ converges uniformly on A.

Next Proposition proves that for every infinite-dimensional Banach space $E$ the space $\left(E, \sigma\left(E, E^{\prime}\right)\right)$ is never a $k$-space.

Proposition 19 If $E$ is a Banach space for which $\left(E, \sigma\left(E, E^{\prime}\right)\right)$ is a $k$-space, then $E$ is finite-dimensional.

Proof Let $\gamma$ be the topology on $E$ of uniform convergence on $\mu\left(E^{\prime}, E\right)$-compact sets. Then clearly $\sigma\left(E, E^{\prime}\right) \leq \gamma$. Since $\sigma\left(E, E^{\prime}\right)$ and $\gamma$ have the same sequentially compact sets by Lemma 5, then the both topologies have the same compact sets (recall that $\sigma\left(E^{\prime}, E\right)$ and $\gamma$ are angelic).

Assume that $\left(E, \sigma\left(E, E^{\prime}\right)\right)$ is a $k$-space, then we have $\sigma\left(E, E^{\prime}\right)=\gamma$. Let $\left(x_{n}\right)_{n}$ be a null-sequence in the norm topology of $E^{\prime}$. Since $\{0\} \cup\left\{x_{n}: n \in \mathbb{N}\right\}$ is $\mu\left(E^{\prime}, E\right)$-compact, then the sequence $\left(x_{n}\right)_{n}$ has finite-dimensional linear span. This yields that $E^{\prime}$ (hence also $E$ ) is finite-dimensional.

\section{Fréchet-Urysohn spaces in class $\mathfrak{G}$}

In this section we prove that every Fréchet-Urysohn as well as every Baire lcs in class $\mathfrak{G}$ is metrizable, see [8]. Recall that a topological space $X$ is Fréchet-Urysohn if for each $A \subset X$ and each $x \in \bar{A}$ there exists a sequence in $A$ which converges to $x$. 
We shall need the following Makarov-type theorem formulated for spaces $E$ in class $\mathfrak{G}$. A sequence of absolutely convex closed subsets $\left(A_{n}\right)_{n}$ in a lcs $E$ is called bornivorous if for every bounded set $B$ in $E$ there exists $A_{n}$ which absorbs $B$.

Lemma 6 Let $E$ be a lcs in class $\mathfrak{G}$ and let $\left\{A_{\alpha}: \alpha \in \mathbb{N}^{\mathbb{N}}\right\}$ be a $\mathfrak{G}$-representation of $E$. For $\alpha=\left(n_{k}\right) \in \mathbb{N}^{\mathbb{N}}$ set

$$
C_{n_{1} \ldots n_{k}}=\bigcup\left\{A_{\beta}: \beta=\left(m_{k}\right) \in \mathbb{N}^{\mathbb{N}}, n_{j}=m_{j}, \quad j=1,2, \ldots k\right\},
$$

for $k \in \mathbb{N}$. Then the polars

$$
C_{n_{1}}^{\circ} \subset C_{n_{1}, n_{2}}^{\circ} \subset \cdots \subset C_{n_{1}, n_{2}, \ldots, n_{k}}^{\circ} \subset \cdots
$$

compose a bornivorous sequence in $E$.

Proof Assume by contradiction that there exists a bounded set $B$ in $E$ such that $B \not \subset k C_{n_{1} \ldots n_{k}}^{\circ}$ for every $k \in \mathbb{N}$. Then for every $k \in \mathbb{N}$ there exists $x_{k} \in B$ such that

$$
k^{-1} x_{k} \notin C_{n_{1} \ldots n_{k}}^{\circ} .
$$

Therefore for every $k \in \mathbb{N}$ there exists $f_{k} \in C_{n_{1} \ldots n_{k}}$ such that

$$
\left|f_{k}\left(k^{-1} x_{k}\right)\right|>1 \text {. }
$$

Then for every $k \in \mathbb{N}$ there exists

$$
\beta_{k}=\left(m_{n}^{k}\right)_{n} \in \mathbb{N}^{\mathbb{N}}
$$

such that $f_{k} \in A_{\beta_{k}}$, where $n_{j}=m_{j}^{k}$ for $j=1,2, \ldots k$. Next define

$$
a_{n}=\max \left\{m_{n}^{k}: k \in \mathbb{N}\right\},
$$

$n \in \mathbb{N}$, and $\gamma=\left(a_{n}\right) \in \mathbb{N}^{\mathbb{N}}$. Since $\gamma \geq \beta_{k}$ for $k \in \mathbb{N}$, then property (b) yields $A_{\beta_{k}} \subset A_{\gamma}$, so $f_{k} \in A_{\gamma}$ for all $k \in \mathbb{N}$. Finally, since by definition any sequence in $A_{\gamma}$ is equicontinuous, one gets that the sequence $\left(f_{k}\right)$ is equicontinuous. Hence the sequence $\left(f_{k}\right)_{k}$ is uniformly bounded on bounded sets in $E$, including $B$, a contradiction.

We need also the following fact from [8].

Lemma 7 For a quasibarrelled space E the following statements are equivalent:

(i) $E$ is in $\mathfrak{G}$;

(ii) There is a family $\mathcal{F}:=\left\{D_{n_{1}, n_{2}, \ldots, n_{k}}: k, n_{1}, n_{2}, \ldots, n_{k} \in \mathbb{N}\right\}$ of absolutely convex closed subsets satisfying

(a) $D_{m_{1}, m_{2}, \ldots, m_{k}} \subset D_{n_{1}, n_{2}, \ldots, n_{k}}$, whenever $n_{i} \leq m_{i}, i=1,2, \ldots, k$;

(b) For every $\alpha=\left(n_{k}\right) \in \mathbb{N}^{\mathbb{N}}$ we have $D_{n_{1}} \subset D_{n_{1}, n_{2}} \subset \cdots \subset D_{n_{1}, n_{2}, \ldots, n_{k}} \subset \cdots$ and the sequence is bornivorous.

(c) If $U_{\alpha}:=\bigcup_{k} D_{n_{1}, n_{2}, \ldots, n_{k}}, \alpha \in \mathbb{N}^{\mathbb{N}}$, then $\left\{U_{\alpha}: \alpha \in \mathbb{N}^{\mathbb{N}}\right\}$ is a basis of neighborhoods of the origin in $E$.

(iii) E has a $\mathfrak{G}$-basis, i.e. a basis of neighborhoods of the origin $\left\{U_{\alpha}: \alpha \in \mathbb{N}^{\mathbb{N}}\right\}$ satisfying the decreasing condition $U_{\beta} \subset U_{\alpha}$ whenever $\alpha \leq \beta$ in $\mathbb{N}^{\mathbb{N}}$.

(iv) The strong dual $\left(E^{\prime}, \beta\left(E^{\prime}, E\right)\right)$ is a quasi-(LB)-space. 
Proof (i) $\Rightarrow$ (ii): Fix a $\mathfrak{G}$-representation $\left\{A_{\alpha}: \alpha \in \mathbb{N}^{\mathbb{N}}\right\}$ of $E$. Since the space $E$ is quasibarrelled, then each set $A_{\alpha}$ is equicontinuous. Hence each set $B_{\alpha}:=A_{\alpha}^{\circ \circ}$ is weakly compact and $\beta\left(E^{\prime}, E\right)$-bounded. This shows that each $B_{\alpha}$ is a $\beta\left(E^{\prime}, E\right)$-Banach disc. Therefore $\left\{B_{\alpha}: \alpha \in \mathbb{N}^{\mathbb{N}}\right\}$ is a resolution consisting of Banach discs in $\left(E^{\prime}, \beta\left(E^{\prime}, E\right)\right)$. This shows that the strong dual $\left(E^{\prime}, \beta\left(E^{\prime}, E\right)\right)$ is a quasi- $(L B)$-space in sense of Valdivia, see [77].

By [77, Proposition 22] there is a resolution $\left\{A_{\alpha}: \alpha \in \mathbb{N}^{\mathbb{N}}\right\}$ in $\left(E^{\prime}, \beta\left(E^{\prime}, E\right)\right)$ consisting of Banach discs with the following property: For every $\beta\left(E^{\prime}, E\right)$-Banach disc $B \subset E^{\prime}$ there is $\alpha \in \mathbb{N}^{\mathbb{N}}$ with $B \subset A_{\alpha}$.

Since each set $A_{\alpha}$ is equicontinuous and $E$ is a quasibarrelled space, then the $\mathfrak{G}$-representation $\left\{A_{\alpha}: \alpha \in \mathbb{N}^{\mathbb{N}}\right\}$ is a fundamental family of equicontinuous subsets of $E^{\prime}$. Hence the family of polars $\left\{A_{\alpha}^{\circ}: \alpha \in \mathbb{N}^{\mathbb{N}}\right\}$ is a basis of neighborhoods of the origin in $E$.

For $k, n_{1}, n_{2}, \ldots, n_{k} \in \mathbb{N}$, we define $C_{n_{1}, n_{2}, \ldots, n_{k}}$ as usual and set

$$
D_{n_{1}, n_{2}, \ldots, n_{k}}:=C_{n_{1}, n_{2}, \ldots, n_{k}}^{\circ} .
$$

For each $\alpha=\left(n_{k}\right) \in \mathbb{N}^{\mathbb{N}}$ we have

$$
V_{\alpha}:={\overline{\bigcup_{k=1}^{\infty} D_{n_{1}, n_{2}, \ldots, n_{k}}}}^{\sigma\left(E, E^{\prime}\right)} \subset\left(\bigcap_{k=1}^{\infty} C_{n_{1}, n_{2}, \ldots, n_{k}}\right)^{\circ} \subset A_{\alpha}^{\circ} .
$$

Since $V_{\alpha}$ is a closed, absolutely convex and bornivorous set, then $V_{\alpha}$ is a neighborhood of zero. By [56, Proposition 8.2.27] one gets for every $\varepsilon>0$

$$
V_{\alpha}=\bigcup_{k=1}^{\infty} D_{n_{1}, n_{2}, \ldots, n_{k}}{ }^{\sigma\left(E, E^{\prime}\right)} \subset(1+\varepsilon) \bigcup_{k=1}^{\infty} D_{n_{1}, n_{2}, \ldots, n_{k}}=(1+\epsilon) U_{\alpha} .
$$

Thus $\left\{U_{\alpha}: \alpha \in \mathbb{N}^{\mathbb{N}}\right\}$ is a basis of $\mathfrak{T}$-neighborhoods of the origin in $E$; let us observe that this prove that (ii) $\Rightarrow$ (iii).

(iii) $\Rightarrow$ (i): The family of polars $\left\{U_{\alpha}^{\circ}: \alpha \in \mathbb{N}^{\mathbb{N}}\right\}$ is a $\mathfrak{G}$-representation of the space $E$.

(iii) $\Rightarrow$ (iv): If $\left\{U_{\alpha}: \alpha \in \mathbb{N}^{\mathbb{N}}\right\}$ is a $\mathfrak{G}$-basis in $E$, then sets $U_{\alpha}^{\circ}$ provide a quasi- $(L B)$-space representation for $\left(E^{\prime}, \beta\left(E^{\prime}, E\right)\right)$.

(iv) $\Rightarrow$ (i): Let $\left\{A_{\alpha}: \alpha \in \mathbb{N}^{\mathbb{N}}\right\}$ be a quasi- $(L B)$ representation for $\left(E^{\prime}, \beta\left(E^{\prime}, E\right)\right)$. Since $E$ is quasibarrelled, then each set $A_{\alpha}$ is equicontinuous. Hence $E$ is in class $\mathfrak{G}$.

Now we are ready to prove Theorem 12, which is due to Cascales, Kạkol and Saxon [8] and generalizes parts of [37, Theorem 2.1] and [50, Theorem 3].

Recall the following concept due to W. Ruess. A lcs $E$ is called b-Baire-like if for every increasing bornivorous sequence $\left(A_{n}\right)_{n}$ of absolutely convex closed subsets of $E$ covering $E$ there exists $n \in \mathbb{N}$ such that $A_{n}$ is a neighbourhood of zero in $E$.

Clearly every locally convex Baire space is Baire-like and every Baire-like space is b-Baire-like.

Theorem 12 For a lcs $E$ in $\mathfrak{G}$ the following statement are equivalent:

(i) E is metrizable.

(ii) $E$ is Fréchet-Uryshon.

(iii) $E$ is b-Baire-like. 
Proof (i) $\Rightarrow$ (ii): is obvious.

(ii) $\Rightarrow$ (iii): Every Fréchet-Urysohn lcs is b-Baire-like [37].

(iii) $\Rightarrow$ (i): If $E$ is b-Baire-like, then $E$ is a quasibarrelled space and therefore we can produce a countable family

$$
\mathcal{F}:=\left\{D_{n_{1}, n_{2}, \ldots, n_{k}}: k, n_{1}, n_{2}, \ldots, n_{k} \in \mathbb{N}\right\}
$$

as in the proof of Lemma 7.

Since the sequence

$$
D_{n_{1}} \subset D_{n_{1}, n_{2}} \subset \cdots \subset D_{n_{1}, n_{2}, \ldots, n_{k}} \subset \cdots
$$

is bornivorous, then for every $\alpha=\left(n_{k}\right) \in \mathbb{N}^{\mathbb{N}}$ we have

$$
E=\bigcup_{k=1}^{\infty} k D_{n_{1}, n_{2}, \ldots, n_{k}}
$$

and, again, since $E$ is b-Baire-like some $D_{n_{1}, n_{2}, \ldots, n_{m}}$ is a neighborhood of the origin for certain $m \in \mathbb{N}$. Thus the family

$$
\mathcal{U}:=\left\{D_{n_{1}, n_{2}, \ldots, n_{k}} \in \mathcal{F}: D_{n_{1}, n_{2}, \ldots, n_{k}} \text { is neighborhood of } 0\right\}
$$

is a countable basis of neighborhoods of the origin for $E$.

This applies to get the following

Corollary 4 Let $\left\{E_{t}: t \in T\right\}$ be a family of lcs. If $T$ is uncountable, then the product $\prod_{t} E_{t}$ is not in class $\mathfrak{G}$.

Proof Assume that $\prod_{t \in T} E_{t}$ is in class $\mathfrak{G}$ and $T$ is an uncountable set. Then $\prod_{t \in T} E_{t}$ contains a subspace of the form $\mathbb{R}^{A}$ for some uncountable set $A$. Clearly $\mathbb{R}^{A}$ is a Baire space in class $\mathfrak{G}$. By Theorem 12 one gets that $\mathbb{R}^{A}$ is metrizable which clearly provides a contradiction.

Lemma 7 provides a large class of lcs in class $\mathfrak{G}$ with a special basis of neighbourhood of zero. Recall that from Fremlin's [59, Theorem 5.5.3] it follows that under Continuum Hypothesis there exists a non-analytic $K$-analytic space $E$ such that each compact set in $E$ is metrizable. On the other hand, we note the following

Example 2 Let $E$ be a nonseparable $(W C G)$ Banach space, for example $E:=c_{0}(\Gamma)$ for uncountable $\Gamma$. Then $\left(E^{\prime}, \sigma\left(E^{\prime}, E\right)\right)$ is $K$-analytic non-analytic with a resolution consisting of nonmetrizable absolutely convex compact sets although every separable compact set in $\left(E^{\prime}, \sigma\left(E^{\prime}, E\right)\right)$ is metrizable.

Indeed, by Theorem 7 the space $\left(E^{\prime}, \sigma\left(E^{\prime}, E\right)\right)$ is $K$-analytic, and since $E$ is nonseparable, then $\left(E^{\prime}, \sigma\left(E^{\prime}, E\right)\right)$ is nonseparable. Since $\left(E, \sigma\left(E^{\prime}, E\right)\right)$ is web-compact, then by [12] every compact separable set in

$$
\left(E^{\prime}, \sigma\left(E^{\prime}, E\right)\right) \subset C_{p}\left(E, \sigma\left(E^{\prime}, E\right)\right)
$$

is metrizable. By Lemma 7 the space $E$ admits a $\mathfrak{G}$-basis

$$
\left\{U_{\alpha}: \alpha \in \mathbb{N}^{\mathbb{N}}\right\} .
$$

Then polar sets $U_{\alpha}^{\circ}$ compose in $\left(E^{\prime}, \sigma\left(E^{\prime}, E\right)\right)$ a resolution consisting of compact absolutely convex sets. Since $E$ is not transeparable, then Proposition 2 applies to get that there exists 
$\alpha_{0} \in \mathbb{N}^{\mathbb{N}}$ such that $U_{\alpha}^{\circ}$ is not metrizable. Set $\mathcal{U}:=\left\{U_{\alpha}^{\circ}: \alpha \geq \alpha_{0}\right\}$. Clearly every $U_{\alpha} \in \mathcal{U}$ is nonmetrizable and $\mathcal{U}$ covers $E^{\prime}$. In fact, if $f \in E^{\prime}$, then there exists a neighbourhood of zero $U$ in $E$ such that $f \in U^{\circ}$. Then there exist $\beta \in \mathbb{N}^{\mathbb{N}}$ and $\gamma \geq \alpha_{0}$ in $\mathbb{N}^{\mathbb{N}}$ such that

$$
U_{\gamma} \subset U_{\alpha_{0}} \cap U_{\beta} \subset U \text {. }
$$

Hence $\mathcal{U}$ is a resolution as required.

\section{Some open problems}

Problem 1 Characterize the strongly realcompacness of $X$ in term of $C(X)$.

Problem 2 When $C_{c}(X)$ is a Baire space, $X$ being a strongly realcompact space?

Problem 3 Characterize for a Fréchet space $E$ the countable tightness of the locally convex space $\left(E^{\prime}, \beta\left(E^{\prime}, E\right)\right)$ in terms of $E$.

Problem 4 Assume that for a lcs in class $\mathfrak{G}$ the tightness of $\left(E, \sigma\left(E, E^{\prime}\right)\right)$ is $\mathfrak{m}$. What topological property in $\left(E^{\prime}, \sigma\left(E^{\prime}, E\right)\right)$ describes this condition?

Problem 5 Describe those lcs $E$ for which there exists a (usco) map from $\mathbb{N}^{\mathbb{N}}$ with compact absolutely convex values covering $E$.

Problem 6 Does there exist a $(D F)$-space not analytic but weakly analytic.

Problem 7 Let $E$ be an analytic $(D F)$-space. Is every bounded set in $E$ a metrizable set?

Problem 8 Is there some corresponding result related with Corollary 3 for spaces $C_{c}(X)$ ?

Problem 9 Describe possible analogue to Proposition 19 for $k_{R}$-property (recall $X$ is a $k_{r^{-}}$space if it is continuous each real function $f$ whose restrictions to each compact subset of $X$ are continuous).

Problem 10 Does 9 admit some generalization for lcs in class $\mathfrak{G}$ ?

Acknowledgments The authors wish to thank the referee for helpful comments and remarks which led to the final form of this paper.

Open Access This article is distributed under the terms of the Creative Commons Attribution Noncommercial License which permits any noncommercial use, distribution, and reproduction in any medium, provided the original author(s) and source are credited.

\section{References}

1. Argyros, S., Mercourakis, S.: On weakly Lindelöf Banach spaces. Rocky Mountain J. Math. 23(2), 395446. doi:10.1216/rmjm/1181072569 (1993)

2. Arkhangel'skii, A. V.: Topological Function Spaces, Mathematics and its Applications, vol. 78, Kluwer, Dordrecht (1992)

3. Batt, J., Hiermeyer, W.: On compactness in $L_{p}(\mu, X)$ in the weak topology and in the topology $\sigma\left(L_{p}(\mu, X), L_{p}\left(\mu, X^{\prime}\right)\right)$. Math. Z. 182, 409-423 (1983)

4. Baumgartner, J.E., van Douwen, E.K.: Strong realcompactness and weakly measurable cardinals. Topol. Appl. 35, 239-251 (1990). doi:10.1016/0166-8641(90)90109-F 
5. Bierstedt, K.D., Bonet, J.: Stefan Heinrich's density condition for Fréchet spaces and the characterization of the distinguished Köthe echelon spaces. Math. Nachr. 35, 149-180 (1988)

6. Cascales, B.: On $K$-analytic locally convex spaces. Arch. Math. 49, 232-244 (1987)

7. Cascales, B., Kạkol, J., Saxon, S.A.: Weight of precompact subsets and tightness. J. Math. Anal. Appl. 269, 500-518 (2002). doi:10.1016/S0022-247X(02)00032-X

8. Cascales, B., Kạkol, J., Saxon, S.A.: Metrizability vs. Fréchet-Urysohn property. Proc. Am. Math. Soc. 131, 3623-3631 (2003)

9. Cascales, B., Namioka, I., Orihuela, J.: The Lindelöf property in Banach spaces. Stud. Math. 154, 165-192 (2003). doi:10.4064/sm154-2-4

10. Cascales, B., Oncina, L.: Compactoid filters and USCO maps. J. Math. Anal. Appl. 282, 826-843 (2003). doi:10.1016/S0022-247X(03)00280-4

11. Cascales, B., Orihuela, J.: On compactness in locally convex spaces, Math. Z. 195(3), 365-381 (1987). doi:10.1007/BF01161762

12. Cascales, B., Orihuela, J.: On pointwise and weak compactness in spaces of continuous functions. Bull. Soc. Math. Belg. Ser. B 40(2), 331-352. Journal continued as Bull. Belg. Math. Soc. Simon Stevin (1988)

13. Diestel, J.: $L_{X}^{1}$ is weakly compactly generated if $X$ is. Proc. Am. Math. Soc. 48(2), 508-510 (1975). doi: $10.2307 / 2040292$

14. van Douwen, E.K.: Prime mappings, number of factors and binary operations. Dissertationes Math. (Rozprawy Mat.) 199, 35 (1981)

15. Drewnowski, L.: Resolutions of topological linear spaces and continuity of linear maps. J. Math. Anal. Appl. 335(2), 1177-1195 (2007). doi:10.1016/j.jmaa.2007.02.032

16. Engelking, R.: General Topology. Heldermann Verlag, Lemgo (1989)

17. Fabian, M., Habala, P., Hájek, P., Montesinos, V., Pelant, J., Zizler, V.: Functional Analysis and InfiniteDimensional Geometry. Canadian Mathematical Society. Springer, Berlin (2001)

18. Ferrando, J.C.: A weakly analytic space which is not $K$-analytic. Bull. Aust. Math. Soc. 79(1), 31-35 (2009). doi:10.1017/S0004972708000968

19. Ferrando, J.C.: Some characterization for $v X$ to be Lindelöf $\Sigma$ or $K$-analytic in term of $C_{p}(X)$. Topol. Appl. 156(4), 823-830 (2009). doi:10.1016/j.topol.2008.10.016

20. Ferrando, J.C., Kąkol, J.: A note on spaces $C_{p}(X) K$-analytic-framed in $\mathbb{R}^{X}$. Bull. Aust. Math. Soc. 78, 141-146 (2008)

21. Ferrando, J.C., Kąkol, J., López-Pellicer, M.: Bounded tightness conditions and spaces $C(X)$. J. Math. Anal. Appl. 297, 518-526 (2004)

22. Ferrando, J.C., Kąkol, J., López-Pellicer, M.: A characterization of trans-separable spaces. Bull. Belg. Math. Soc. Simon Stevin 14, 493-498 (2007)

23. Ferrando, J.C., Ka̧kol, J., López-Pellicer, M.: Metrizability of precompact sets: an elementary proof. Rev. R. Acad. Cienc. Exactas Fis. Nat. Ser. A. Mat. RACSAM 99(2), 135-142 (2005). http://www.rac.es/ ficheros/doc/00173.pdf

24. Ferrando, J.C., Kạkol, J., López-Pellicer, M., Saxon, S.A.: Tightness and distinguished Fréchet spaces. J. Math. Anal. Appl. 324, 862-881 (2006). doi:10.1016/j.jmaa.2005.12.059

25. Ferrando, J.C., Kąkol, J., López-Pellicer, M., Saxon, S.A.: Quasi-Suslin weak duals. J. Math. Anal. Appl. 339(2), 1253-1263 (2008). doi:10.1016/j.jmaa.2007.07.081

26. Floret, K.: Weakly compact sets. Lecture Notes in Mathematics, vol. 801, Springer, Berlin (1980)

27. Gillman, L., Henriksen, M.: Rings of continuous functions in which every finitely generated ideal is principial. Trans. Am. Math. Soc. 82, 366-391 (1956). doi:10.2307/1993054

28. Gillman, L., Jerison, M.: Rings of Continuous Functions. Van Nostrand Reinhold Company, New York (1960)

29. Grothendieck, A.: Sur les applications linéaires faiblement compactes d'espaces du type $C(K)$. Can. J. Math. 5, 129-173 (1953)

30. Gullick, D., Schmets, J.: Separability and semi-norm separability for spaces of bounded continuous functions. Bull. R. Sci. Lige 41, 254-260 (1972)

31. Hager, A.W.: Some nearly fine uniform spaces. Proc. Lond. Math. Soc. 28, 517-546 (1974). doi:10.1112/ $\mathrm{plms} / \mathrm{s} 3-28.3 .517$

32. Howes, N.R.: On completeness. Pacific J. Math. 38, 431-440 (1971)

33. Isbell, J.R.: Uniform spaces. In: Mathematical Surveys 12, American Mathematical Society, Providence (1964)

34. Kąkol, J., López-Pellicer, M.: Compact coverings for Baire locally convex spaces. J. Math. Anal. Appl. 332, 965-974 (2007). doi:10.1016/j.jmaa.2006.10.045

35. Kąkol, J., López-Pellicer, M.: A characterization of Lindelöf $\Sigma$-spaces $v X$ (preprint) 
36. Kąkol, J., López-Pellicer, M., Sliwa, W.: Weakly $K$-analytic spaces and the three-space property for analyticity. J. Math. Anal. Appl. 362(1), 90-99 (2010). doi:10.1016/j.jmaa.2009.09.026

37. Kąkol, J., Saxon, S.: Montel $(D F)$-spaces, sequential $(L M)$-spaces and the strongest locally convex topology. J. Lond. Math. Soc. 66(2), 388-406 (2002)

38. Ka̧kol, J., Saxon, S., Todd, A.T.: Pseudocompact spaces $X$ and $d f$-spaces $C_{c}(X)$. Proc. Am. Math. Soc. 132, 1703-1712 (2004)

39. Kąkol, J., Śliwa, W.: Strongly Hewitt spaces. Topology Appl. 119(2), 219-227 (2002). doi:10.1016/ S0166-8641(01)00063-3

40. Khan, L.A.: Trans-separability in spaces of continuous vector-valued functions. Demonstr. Math. 37, 6167 (2004)

41. Khan, L.A.: Trans-separability in the strict and compact-open topologies. Bull. Korean Math. Soc. 45, 681-687 (2008). doi:10.4134/BKMS.2008.45.4.681

42. Khurana, S.S.: Weakly compactly generated Fréchet spaces. Int. J. Math. Math. Sci. 2(4), 721-724 (1979). doi:10.1155/S0161171279000557

43. Kirk, R.B.: A note on the Mackey topology for $\left(C^{b}(X)^{*}, C^{b}(X)\right)$. Pacific J. Math. 45(2), 543-554 (1973)

44. Köthe, G.: Topological Vector Spaces I. Springer, Berlin (1969)

45. Kubiś, W., Okunev, O., Szeptycki, P.J.: On some classes of Lindelöf $\Sigma$-spaces. Topol. Appl. 153(14), 2574-2590 (2006). doi:10.1016/j.topol.2005.09.009

46. Künzi, H.P.A., Mršević, M., Reilly, I.L., Vamanamurthy, M.K.: Pre-Lindelöf quasi-pseudo-metric and quasi-uniform spaces. Mat. Vesnik 46, 81-87 (1994)

47. Megginson, R.: An Introduction to Banach Space Theory. Springer, Berlin (1988)

48. Michael, E.: $\aleph_{0}$-spaces. J. Math. Mech. 15, 983-1002 (1966)

49. Nagami, K.: $\Sigma$-spaces. Fund. Math. 61, 169-192 (1969)

50. Narayanaswami, P.P., Saxon, S.A.: (LF)-spaces, quasi-Baire spaces and the strongest locally convex topology. Math. Ann. 274, 627-641 (1986). doi:10.1007/BF01458598

51. Negrepontis, S.: Absolute Baire sets. Proc. Am. Math. Soc. 18(4), 691-694 (1967). doi:10.2307/2035440

52. Orihuela, J.: Pointwise compactness in spaces of continuous functions. J. Lond. Math. Soc. 36(2), 143-152 (1987). doi:10.1112/jlms/s2-36.1.143

53. Orihuela, J.: On weakly Lindelöf Banach spaces. In: Bierstedt, K.D. et al. (eds.) Progress in Functional Analysis, pp. 279-291. Elsvier, Amsterdam (1992). doi:10.1016/S0304-0208(08)70326-8

54. Orihuela, J., Schachermayer, W., Valdivia, M.: Every Readom-Nikodym Corson compact space is Eberlein compact. Stud. Math. 98, 157-174 (1992)

55. Orihuela, J., Valdivia, M.: Projective generators and resolutions of identity in Banach spaces. Rev. Mat. Complut. 2(Supplementary Issue), 179-199 (1989)

56. Pérez Carreras, P., Bonet, J.: Barrelled Locally Convex Spaces, Mathematics Studies 131. North-Holland, Amsterdam (1987)

57. Pfister, H.H.: Bemerkungen zum Satz über die separabilität der Fréchet-Montel Raüme. Arch. Math. (Basel) 27, 86-92 (1976). doi:10.1007/BF01224645

58. Robertson, N.: The metrisability of precompact sets. Bull. Aust. Math. Soc. 43(1), 131-135 (1991). doi: $10.1017 /$ S0004972700028847

59. Rogers, C.A., Jayne, J.E., Dellacherie, C., Topsøe, F., Hoffman-Jørgensen, J., Martin, D.A., Kechris, A.S., Stone, A.H.: Analytic Sets. Academic Press, London (1980)

60. Saxon, S.A.: Nuclear and product spaces, Baire-like spaces, and the strongest locally convex topology. Math. Ann. 197(2), 87-106 (1972). doi:10.1007/BF01419586

61. Schawartz, L.: Radom Measures on Arbitrary Topological Spaces and Cylindrical Measures. Oxford University Press, Oxford (1973)

62. Schlüchtermann, G., Wheller, R.F.: On strongly WCG Banach spaces. Math. Z. 199(3), 387-398 (1988). doi:10.1007/BF01159786

63. Schlüchtermann, G., Wheller, R.F.: The Mackey dual of a Banach space. Note Math. 11, 273-287 (1991)

64. Schmets, J.: Espaces de functions continues. Lecture Notes in Mathematics, vol 519, Springer-Verlag, Berlin-New York (1976)

65. Talagrand, M.: Sur une conjecture de H. H. Corson. Bull. Soc. Math. 99, 211-212 (1975)

66. Talagrand, M.: Espaces de Banach faiblement $K$-analytiques. Ann. Math. 110(2), 407-438 (1979)

67. Talagrand, M.: Weak Cauchy sequences in $L^{1}(E)$. Am. J. Math. 106(3), 703-724 (1984). doi:10.2307/ 2374292

68. Tkachuk, V.V.: A space $C_{p}(X)$ is dominated by irrationals if and only if it is $K$-analytic. Acta Math. Hungar. 107(4), 253-265 (2005)

69. Tkachuk, V.V.: Lindelöf $\Sigma$-spaces: an omnipresent class. RACSAM Rev. R. Acad. Cienc. Exactas Fis. Nat. Ser. A. Mat. 104,(2), 221-244 (2010). doi:10.5052/RACSAM.2010.15 
70. Todd, A.R., Render, H.: Continuous function spaces, (db)-spaces and strongly Hewitt spaces. Topol. Appl. 141, 171-186 (2004). doi:10.1016/j.topol.2003.12.005

71. Valdivia, M.: Topics in Locally Convex Spaces, Mathematics Studies 67. North-Holland, Amsterdam (1982)

72. Valdivia, M.: Espacios de Fréchet de generación débilmente compacta. Collect. Math. 38, 17-25 (1987)

73. Valdivia, M.: Resolutions of identity in certain Banach spaces. Collect. Math. 38, 124-140 (1988)

74. Valdivia, M.: Resolutions of identity in certain metrizable locally convex spaces. Rev. R. Acad. Cienc. Exactas Fis. Nat. (Esp.) 83, 75-96 (1989)

75. Valdivia, M.: Projective resolutions of identity in $C(K)$ spaces. Arch. Math. (Basel) 54, 493-498 (1990)

76. Valdivia, M.: Resoluciones proyectivas del operador identidad y bases de Markusevich en ciertos espacios de Banach. Rev. R. Acad. Cienc. Exactas Fis. Nat. (Esp.) 84, 23-34

77. Valdivia, M.: Quasi-LB-spaces. J. Lond. Math. Soc. 35(2), 149-168 (1987). doi:10.1112/jlms/s2-35.1. 149

78. Walker, R.C.: The Stone-Čech compactification Ergebnisse der Mathematik und ihrer Grenzgebiete. Band 83. Springer, Berlin (1974) 